\title{
Effective synthesis of novel furan-fused pentacyclic triterpenoids via anionic 5-exo dig cyclization of 2-alkynyl-3-oxotriterpene acids
}

\author{
Rinat R. Gubaidullin, Darina S. Yarmukhametova, \\ Darya A. Nedopekina, Rezeda R. Khalitova, and Anna Yu. Spivak* \\ Institute of Petrochemistry and Catalysis, Russian Academy of Sciences, \\ 141 Prospekt Oktyabrya, Ufa 450 075, Russian Federation \\ E-mail:spivak.ink@gmail.com
}

Received 04-20-2017

Accepted 06-28-2017

Published on line $07-23-2017$

\section{Abstract}

An efficient synthetic route to biologically interesting furan-fused pentacyclic triterpenoids with a furan moiety 2,3-annelated to the terpenoid skeleton has been developed. New heterocyclic triterpenoids have been obtained in moderate to good yields by base-promoted 5-exo-dig cyclization of the pent-4-yn-1-one moiety in ring $A$ of the 2-alkynyl-3-oxotriterpene acids of lupane, ursane and oleane type.

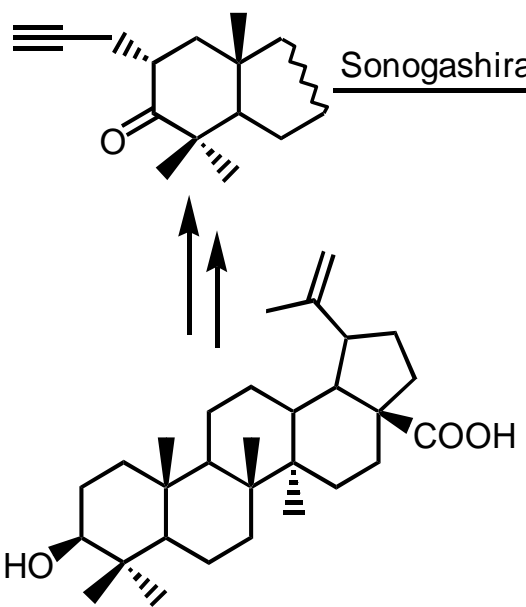

Betulinic acid

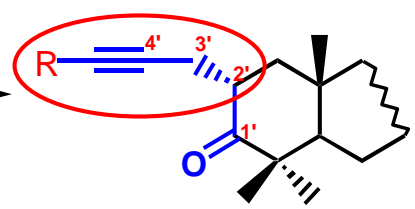

1. $\mathrm{KN}\left(\mathrm{SiMe}_{3}\right)_{2}, \mathrm{DME}, 20^{\circ} \mathrm{C}$

2. Lil, DMF, reflux

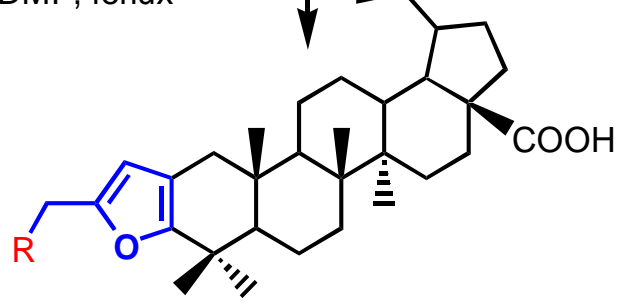

10 examples

Keywords: Pentacyclic triterpenoids, heterocycles, furans, 4-pentynones, 5-exo-dig cyclisation 


\section{Introduction}

Pentacyclic triterpenoids, which are abundant in plants, are usually produced as secondary metabolites. These compounds are of interest for pharmacological investigations, as they exhibit a variety of biological activities, including anti-inflammatory, antiviral, hepatoprotective, antiparasitic, and, what is important, anticancer activities, which are successfully combined with low systemic toxicity for animals. ${ }^{1-8}$ Owing to the presence of easily transformable functional groups $(\mathrm{OH}, \mathrm{COOH}, \mathrm{C}=\mathrm{C})$, pentacyclic triterpenoids possess a good synthetic potential and are actively used as promising structural platforms for the discovery of new drugs. Currently, in order to increase the biological potential and bioavailability of native triterpene acids, their numerous synthetic analogues have been prepared. Among them, considerable attention has been given to heterocyclic triterpenoids with various nitrogen, sulfur, and oxygen heterocycles. ${ }^{9-18}$ The biological activity of triterpenoid heterocycles are promising and many of them have been studied as antitumor, ${ }^{9-13}$ antiosteoporosis, ${ }^{14,15}$ antiinflammatory ${ }^{16}$ and antileishmanial agents. ${ }^{17,18}$ Among this group of potentially biologically active compounds, furan triterpenoid derivatives have not been reported in the literature. Meanwhile, polysubstituted furans represent an important class of oxygen heterocycles and occur as structural moieties in many natural products and pharmaceutically important substances. ${ }^{19}$ Furans are used in medicinal chemistry as useful intermediates in the synthetic transformations aimed at the development of new pharmaceutical agents. ${ }^{20} \mathrm{~A}$ recent trend in the synthesis of polysubstituted furans is related to the development of atom-economic methods for the design of the furan ring via intramolecular cyclization of acyclic alkynyl ketones or alcohols induced by strong bases $^{21-23}$ or transition metal-based catalysts. ${ }^{24-27}$ These rational methods that give furan heterocycles under mild conditions provide a good alternative to numerous classical methods for furan synthesis, in particular known cyclocondensations of carbonyl compounds (Paal-Knorr or Feist-Benary syntheses).

Recently we developed a chemoselective method for the synthesis of $C(2)$-propargyl-substituted lupaneand ursane-type triterpenoids based on $\alpha$-alkylation with propargyl bromide of potassium enoxytriethylborates generated from 3-oxotriterpenes under the action of $\mathrm{KN}\left(\mathrm{SiMe}_{3}\right)_{2}-\mathrm{Et}_{3} \mathrm{~B} .{ }^{28}$

In this work, the C-2 alkynyl derivatives of 3-oxotriterpene acids were used as the key intermediates for the synthesis of new furanotriterpenoids by anionic 5-exo-dig cyclization of the pent-4-yn-1-one moiety in the A-ring of the pentacyclic skeleton. A specific feature of this transformation is that the pent-4-yn-1-one moiety is incorporated in polycyclic molecules, structurally related to steroids, whereas in many cases, acyclic alkynyl ketones have been used as the initial substrates for cyclization into furans. ${ }^{21-27}$ To our knowledge, only one research group has described the synthesis of $[3,2-b]$ furan-fused steroids through anionic annulation reaction of 4-pentynone moiety on the A-ring of a steroid core. ${ }^{29}$

\section{Results and Discussion}

The initial compounds $\mathbf{8 - 1 0}$ were synthesized by a reported method ${ }^{28}$ via the reaction of propargyl bromide with the enolate anion, which was formed by treating the methyl esters of betulonic 2, ursonic 4, and oleanonic acids 6 with $\mathrm{KN}\left(\mathrm{SiMe}_{3}\right)_{2}-\mathrm{Et}_{3} \mathrm{~B}$ in 1,2-dimethoxyethane at room temperature. The reactions afforded $C(2)$-propargyl triterpene acid derivatives 8-10 with equatorial-oriented $\alpha$-propynyl groups (Scheme 1). 


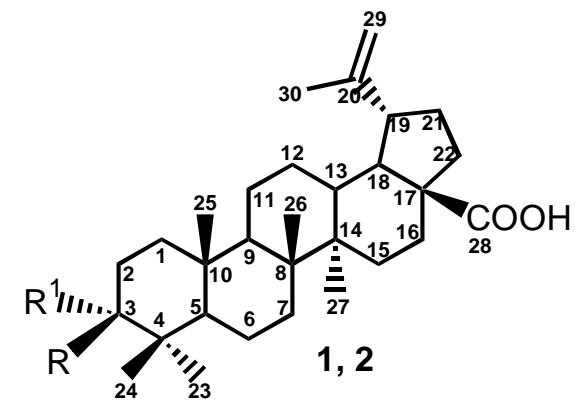

$\mathrm{R}=\mathrm{OH}, \mathrm{R}^{1}=\mathrm{H}$ Betulinic acid $\mathbf{1}$

$\mathrm{R}+\mathrm{R}^{1}=\mathrm{O} \quad$ Betulonic acid 2

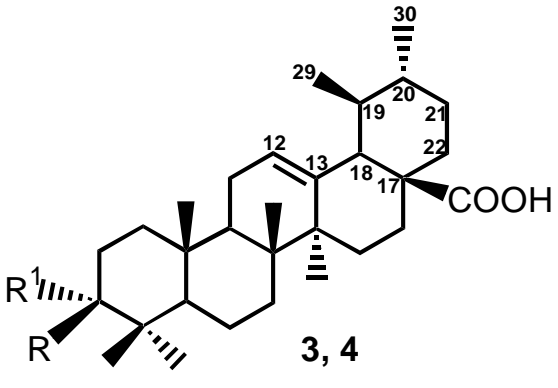

$\mathrm{R}=\mathrm{OH}, \mathrm{R}^{1}=\mathrm{H} \quad$ Ursolic acid 3 $\mathrm{R}+\mathrm{R}^{1}=\mathrm{O} \quad$ Ursonic acid 4

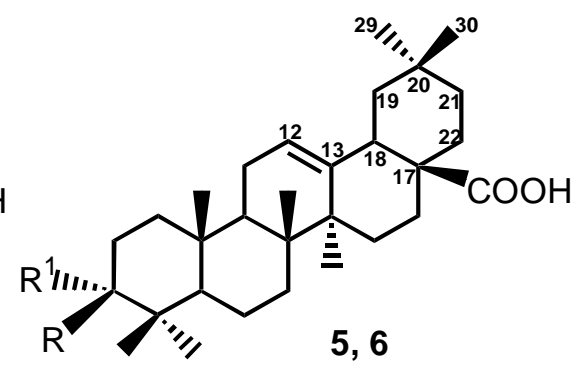

$\mathrm{R}=\mathrm{OH}, \mathrm{R}^{1}=\mathrm{H}$ Oleanolic acid $\mathbf{5}$

$R+R^{1}=0 \quad$ Oleanonic acid 6

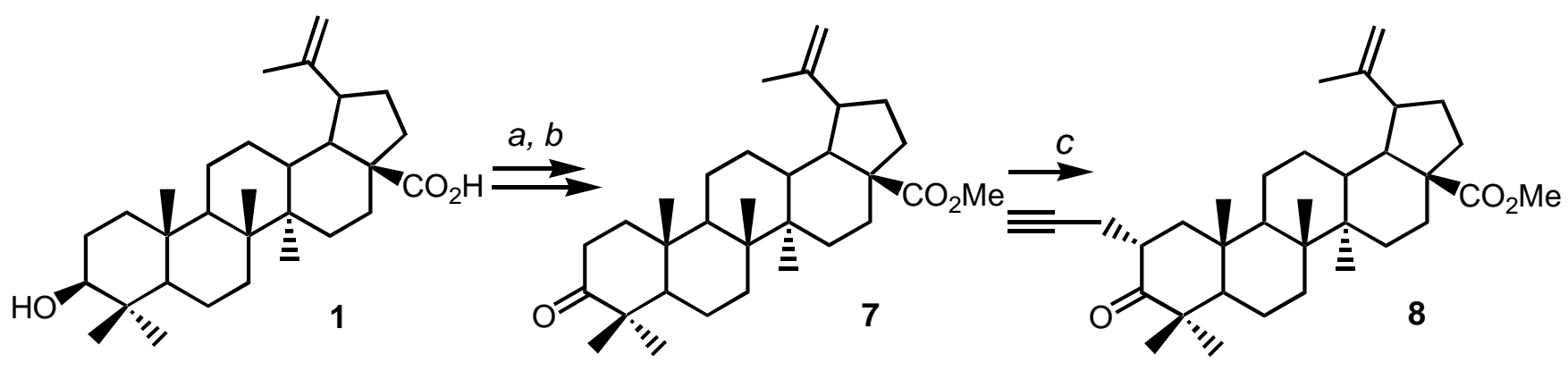

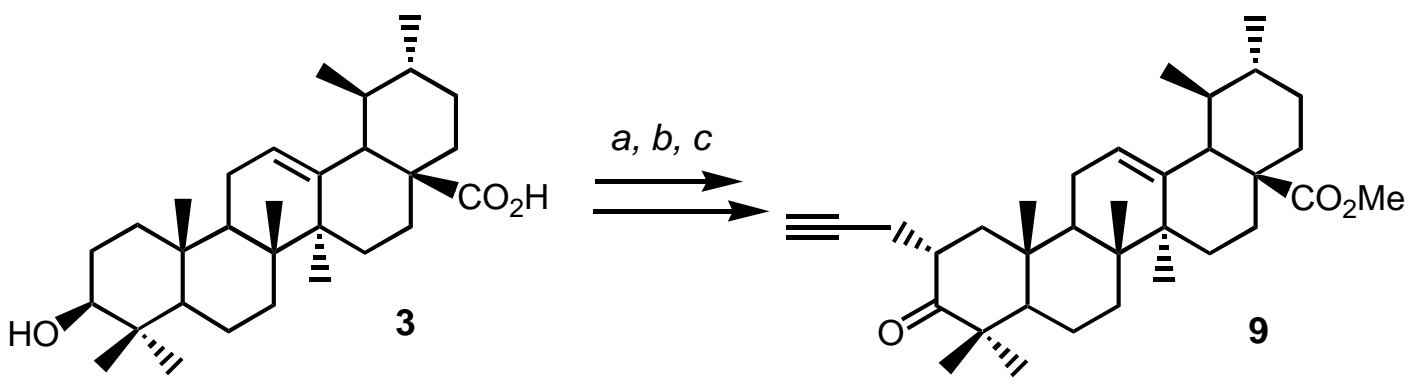<smiles>CC1(C)CC[C@]2(C(=O)O)CC[C@]3(C)C(=CCC4C3CC[C@@]3(C)C(C)(C)[C@@H](O)CC[C@]43C)C2C1</smiles>

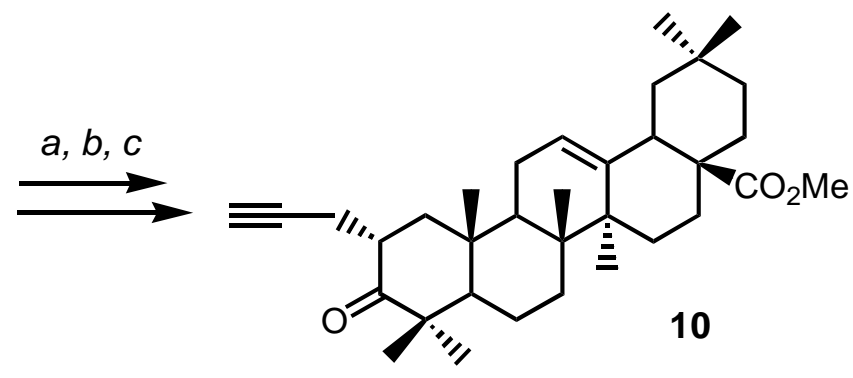

Reagents and conditions: $a, \mathrm{CrO}_{3}, \mathrm{H}_{2} \mathrm{SO}_{4}$, acetone, or $\mathrm{PCC}, \mathrm{CH}_{2} \mathrm{Cl}_{2} ; \quad b, \mathrm{CH}_{2} \mathrm{~N}_{2}, \mathrm{Et}_{2} \mathrm{O}$; $c, \mathrm{KN}\left(\mathrm{SiMe}_{3}\right)_{2}-\mathrm{Et}_{3} \mathrm{~B}, \mathrm{DME}$.

Scheme 1. The preparation of C-2 propargyl triterpene acid derivatives 8-10.

The cyclization conditions were selected in relation to lupane triterpenoid 8. Upon the reaction with superbases, $^{30}$ BuOK $^{\mathrm{t}}-\mathrm{DMSO}, \mathrm{BuOK}^{\mathrm{t}}-\mathrm{DMF}, \mathrm{BuOK}^{\mathrm{t}}-\mathrm{DME}, \mathrm{KOH}-\mathrm{THF}$, or $\mathrm{KOH}-\mathrm{DMSO}$, at room temperature over a period of 1-2 h, compound 8 was fully converted into a complex mixture of oligomeric compounds, in which the desired product 11a was not found. The use of $\mathrm{KN}\left(\mathrm{SiMe}_{3}\right)_{2}$ in DMSO gave heterocycle 11a in a yield not exceeding $36 \%$. The expected compound 11a was obtained in a reasonable yield of $58 \%$ by treatment of terpenoid 8 with $\mathrm{KN}\left(\mathrm{SiMe}_{3}\right)_{2}$ in dimethoxyethane at room temperature for $30 \mathrm{~min}$. With longer reaction times, the yield of furan derivative 11a was lower as a result of formation of oligomeric side products. Under the 
optimized conditions, methyl ursonate $\mathbf{9}$ and methyl oleonate $\mathbf{1 0}$ were converted to heterocyclic compounds $12 \mathrm{a}$ and $13 \mathrm{a}$ in $56 \%$ and $54 \%$ yields, respectively (Scheme 2, Table 1 ).

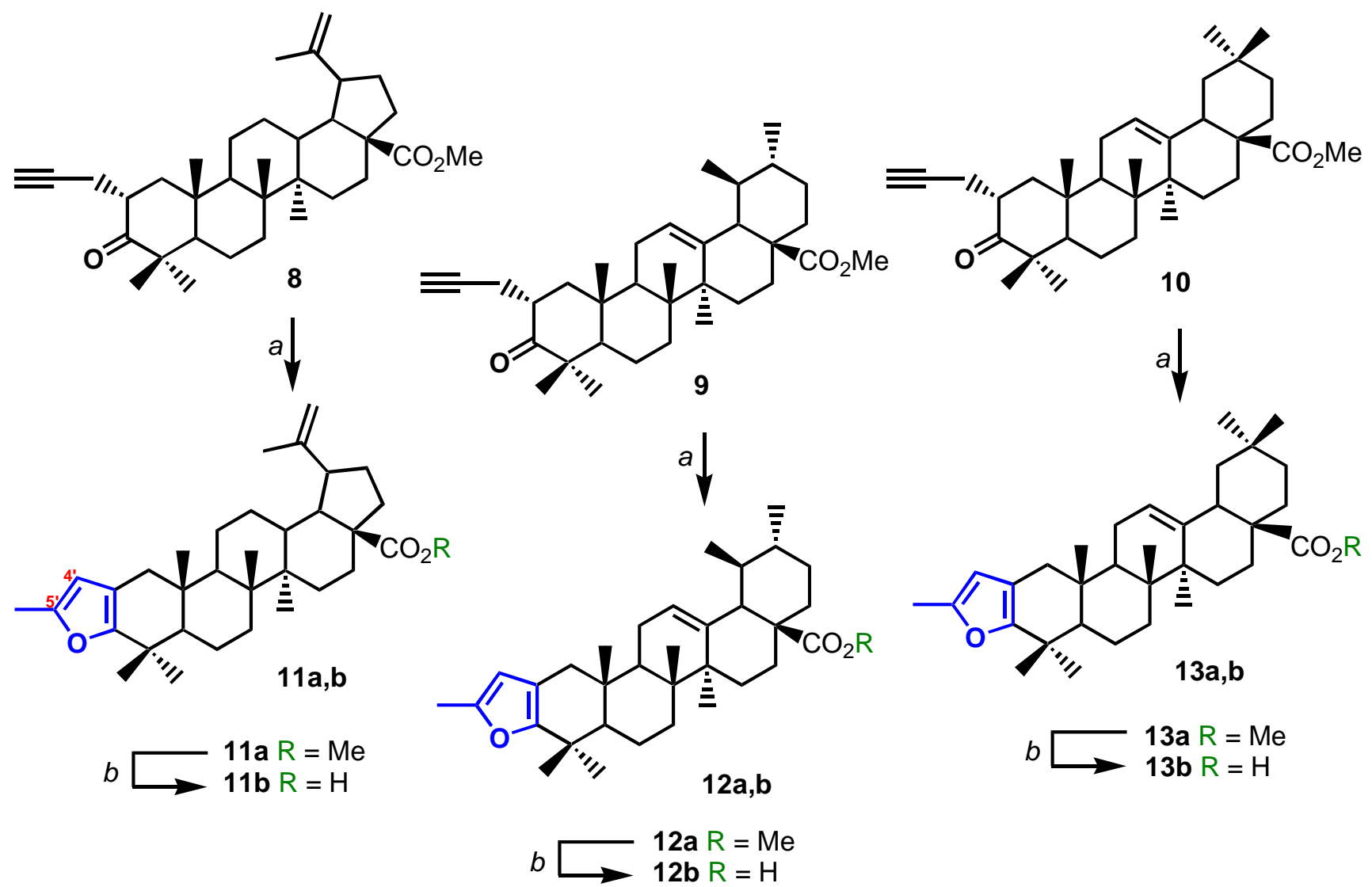

Reagents and conditions: $a, \mathrm{KN}\left(\mathrm{SiMe}_{3}\right)_{2}-\mathrm{Et}_{3} \mathrm{~B}, \mathrm{DME}, \mathrm{rt}, \mathrm{Ar} ; \quad b$, Lil, DMF, reflux, $\mathrm{Ar}$

Scheme 2. Synthesis of [3,2-b]furan fused triterpenoids $\mathbf{1 1 a , b}, \mathbf{1 2} \mathbf{a}, \mathbf{b}$ and $\mathbf{1 3} \mathbf{a}, \mathbf{b}$.

Demethylation of the sterically hindered ester group in compounds 11a-13a via halideolysis with Lil in $\mathrm{DMF}^{28}$ afforded $\mathbf{1 1 b - 1 3 b}$ in $54-56 \%$ yield (Scheme 2 ).

In order to broaden the applicability of this method, aryl groups with various substituents in the aromatic ring $\left(4-\mathrm{Cl}, 4-\mathrm{Br}, 4-\mathrm{F}, 3,4,5-(\mathrm{OMe})_{3}, 2-\mathrm{Me}, 4-\mathrm{NO}_{2}\right)$ were introduced into the terminal acetylenic moiety of compounds 8-10. These products were obtained in excellent yield (80-85\%) by the Sonogashira reaction in the presence of $\mathrm{PdCl}_{2}\left(\mathrm{PPh}_{3}\right)_{2}$, Cul and $\mathrm{Et}_{3} \mathrm{~N}$ (Scheme 3).

The resulting alkynyl triterpenoid derivatives $14 \mathrm{a}-\mathrm{g}, \mathbf{1 7}$, and 19 were successfully converted into triterpene furans 15a-g, 18a and 20a. Cleavage of methyl esters afforded compounds 16a-f, 18b and 20b. Triterpenoids with arylalkynyl substituents were more reactive in this intramolecular cyclization than the substrates containing a terminal acetylenic bond. As opposed to cyclization of triterpenoid $\mathbf{8}$, compound 15a was transformed into furan derivative 15a in a good yield (54-73\%) in the presence of various basic reagents: $\mathrm{BuOK}^{\mathrm{t}}-\mathrm{DMSO}, \mathrm{KN}\left(\mathrm{SiMe}_{3}\right)_{2}$-DMSO, BuOK ${ }^{\mathrm{t}}-\mathrm{DME}$ or $\mathrm{KN}\left(\mathrm{SiMe}_{3}\right)_{2}-\mathrm{DME}$. However the best yield of furan derivative 14a (73\%) was obtained with the $\mathrm{KN}\left(\mathrm{SiMe}_{3}\right)_{2}$-DME (Table 1). Among the tested 2-arylacetylenic derivatives of betulonic acid 14a-g only triterpenoid $14 \mathrm{~g}\left(\mathrm{R}=4-\mathrm{O}_{2} \mathrm{NC}_{6} \mathrm{H}_{4}\right)$ was a problematic compound. Intramolecular cyclisation provided a $19 \%$ isolation yield of furanoterpenoid $15 \mathrm{~g}$ and led to the formation of large amounts of side-products. 
Aryl-substituted acetylene derivatives 14a-g, 17, and 19 were cycloisomerized on treatment with $\mathrm{KN}\left(\mathrm{SiMe}_{3}\right)_{2}$-DME markedly faster (in 10-12 $\mathrm{min}$ ) than propargyl-substituted triterpenoids 8-10 (30 min) to give the target reaction products 15a-g, 18a, and 20a in higher yields (70-77\%).

Table 1. Reaction conditions for the synthesis of $[3,2-b]$ furan-fused triterpenoids

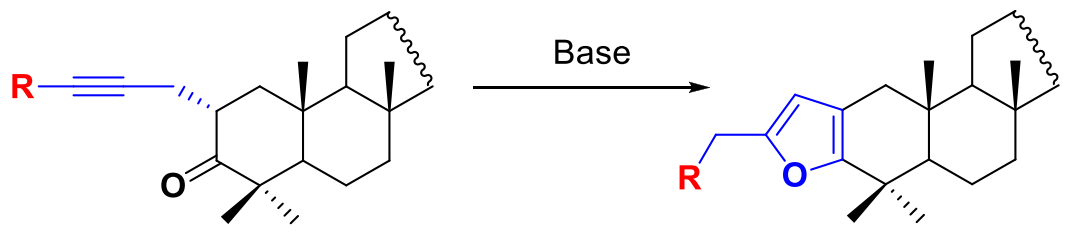

$8-10,14 a-g, 17$ and 19

11a-13a, 15a-g, 18a and 20a

\begin{tabular}{|c|c|c|c|c|c|c|}
\hline Entry & $\begin{array}{c}\text { C-2-alkynyl } \\
\text { triterpenoids }\end{array}$ & $\mathrm{R}$ & Base & Solvent & $\begin{array}{c}{[3,2-b] \text { furan-fused }} \\
\text { triterpenoids }\end{array}$ & $\begin{array}{c}\text { Yield }^{a} \\
\%\end{array}$ \\
\hline 1 & 8 & $\mathrm{H}$ & $\mathrm{BuOK}^{\mathrm{t}}$ & DMSO & $11 a$ & 0 \\
\hline 2 & 8 & $\mathrm{H}$ & $\mathrm{BuOK}^{\mathrm{t}}$ & DMF & $11 a$ & 0 \\
\hline 3 & 8 & $\mathrm{H}$ & $\mathrm{BuOK}^{\mathrm{t}}$ & DME & $11 a$ & 0 \\
\hline 4 & 8 & $\mathrm{H}$ & $\mathrm{KOH}$ & THF & $11 a$ & 0 \\
\hline 5 & 8 & $\mathrm{H}$ & $\mathrm{KOH}$ & DMSO & $11 a$ & 0 \\
\hline 6 & 8 & $\mathrm{H}$ & $\mathrm{KN}\left(\mathrm{SiMe}_{3}\right)_{2}$ & DMSO & $11 a$ & 36 \\
\hline 7 & 8 & $\mathrm{H}$ & $\mathrm{KN}\left(\mathrm{SiMe}_{3}\right)_{2}$ & DME & $11 a$ & 58 \\
\hline 8 & 9 & $\mathrm{H}$ & $\mathrm{KN}\left(\mathrm{SiMe}_{3}\right)_{2}$ & DME & $12 a$ & 56 \\
\hline 9 & 10 & $\mathrm{H}$ & $\mathrm{KN}\left(\mathrm{SiMe}_{3}\right)_{2}$ & DME & $13 a$ & 54 \\
\hline 10 & $14 a$ & $\mathrm{Ph}$ & $\mathrm{BuOK}^{\mathrm{t}}$ & DMSO & $15 a$ & 54 \\
\hline 11 & $14 a$ & $\mathrm{Ph}$ & $\mathrm{BuOK}^{\mathrm{t}}$ & DME & $15 a$ & 70 \\
\hline 12 & $14 a$ & $\mathrm{Ph}$ & $\mathrm{KN}\left(\mathrm{SiMe}_{3}\right)_{2}$ & DMSO & $15 a$ & 57 \\
\hline 13 & $14 a$ & $\mathrm{Ph}$ & $\mathrm{KN}\left(\mathrm{SiMe}_{3}\right)_{2}$ & DME & $15 a$ & 73 \\
\hline 14 & $14 b$ & $4-\mathrm{BrC}_{6} \mathrm{H}_{4}$ & $\mathrm{KN}\left(\mathrm{SiMe}_{3}\right)_{2}$ & DME & $15 b$ & 71 \\
\hline 15 & $14 c$ & $4-\mathrm{ClC}_{6} \mathrm{H}_{4}$ & $\mathrm{KN}\left(\mathrm{SiMe}_{3}\right)_{2}$ & DME & $15 c$ & 70 \\
\hline 16 & $14 d$ & $4-\mathrm{FC}_{6} \mathrm{H}_{4}$ & $\mathrm{KN}\left(\mathrm{SiMe}_{3}\right)_{2}$ & DME & $15 d$ & 72 \\
\hline 17 & $14 \mathrm{e}$ & $2-\mathrm{CH}_{3} \mathrm{C}_{6} \mathrm{H}_{4}$ & $\mathrm{KN}\left(\mathrm{SiMe}_{3}\right)_{2}$ & DME & $15 e$ & 77 \\
\hline 18 & $14 f$ & $3,4,5-\left(\mathrm{CH}_{3} \mathrm{O}\right)_{3} \mathrm{C}_{6} \mathrm{H}_{2}$ & $\mathrm{KN}\left(\mathrm{SiMe}_{3}\right)_{2}$ & DME & $15 f$ & 73 \\
\hline 19 & $14 \mathrm{~g}$ & $4-\mathrm{O}_{2} \mathrm{NC}_{6} \mathrm{H}_{4}$ & $\mathrm{KN}\left(\mathrm{SiMe}_{3}\right)_{2}$ & DME & $15 \mathrm{~g}$ & 19 \\
\hline 20 & 17 & $\mathrm{Ph}$ & $\mathrm{KN}\left(\mathrm{SiMe}_{3}\right)_{2}$ & DME & $18 a$ & 71 \\
\hline 21 & 19 & $\mathrm{Ph}$ & $\mathrm{KN}\left(\mathrm{SiMe}_{3}\right)_{2}$ & DME & $20 a$ & 72 \\
\hline
\end{tabular}

a Yield of isolated product.

The structures of all new compounds were confirmed by conventional analytical methods. The ${ }^{1} \mathrm{H}$ and ${ }^{13} \mathrm{C}$ NMR spectra of furan-fused triterpenoids adequately reflected their structure. Indeed, the ${ }^{13} \mathrm{C}$ NMR spectrum of compound 11a exhibited no signals for the acetylene and carbonyl carbon atoms, indicating that these functional groups of the initial methyl betulonate $\mathbf{8}$ were transformed in the intramolecular cyclization. Apart from the characteristic signal for the quaternary C-20 carbon atom (150.57 ppm), the spectrum exhibited 
three new signals for quaternary carbon atoms (DEPT, HSQC) at 113.68, 149.57, and 154.44 ppm, which were assigned to $\mathrm{C}-2, \mathrm{C}-5^{\prime}$, and $\mathrm{C}-3$, respectively. The ${ }^{1} \mathrm{H}$ NMR spectrum contained, apart from the signals for protons at $\mathrm{C}-29$, a new singlet for the vinylic $\mathrm{H}-4^{\prime}$ proton at about $5.68 \mathrm{ppm}$. The proton signals for the methyl group at the furan $\mathrm{C}^{-5^{\prime}}$ atom occurred at $2.26 \mathrm{ppm}$. The (Me)C-5' carbon atom resonated at $13.71 \mathrm{ppm}$. The spectroscopic data indicated the presence of tetrasubstituted C-2-C-3 and trisubstituted C $-4^{\prime}-\mathrm{C}_{-} 5^{\prime}$ double bonds in compound 11a.
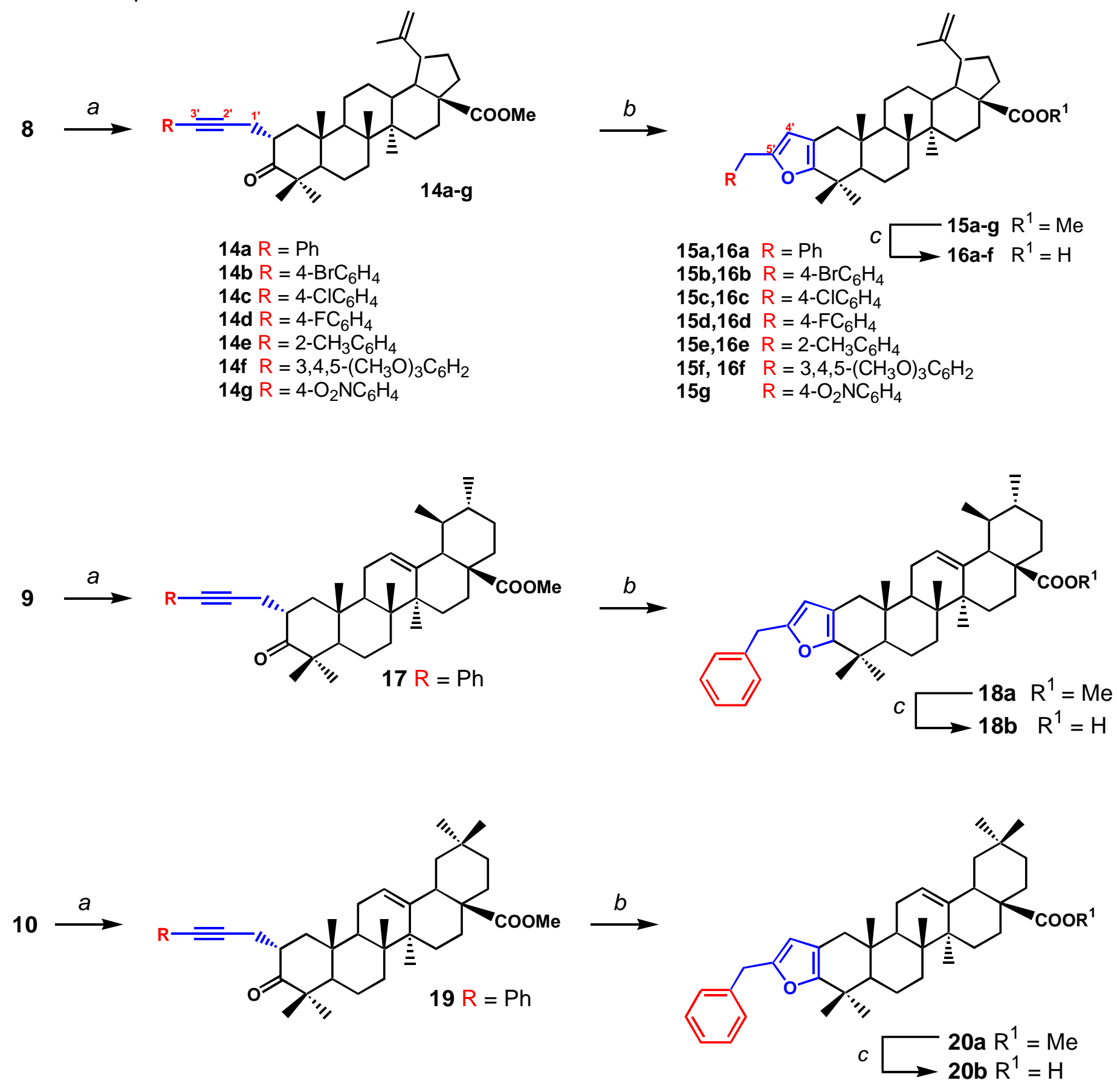

Reagents and conditions: a, $\mathrm{Arl}, \mathrm{PdCl}_{2}\left(\mathrm{PPh}_{3}\right)_{2}, \mathrm{Cul}, \mathrm{Et}_{3} \mathrm{~N}, \mathrm{DMF}, \mathrm{Ar}, 20{ }^{\circ} \mathrm{C}$;

b, $\mathrm{KN}\left(\mathrm{SiMe}_{3}\right)_{2}, \mathrm{DME}, 20^{\circ} \mathrm{C}$ or BuOK ${ }^{\dagger}, \mathrm{DME}, 20^{\circ} \mathrm{C}$. C, Lil, DMF, reflux, Ar.

Scheme 3. Synthesis of [3,2-b]furan fused triterpenoids $15 a-g, 16 a-f, 18 a, b$ and $20 a, b$. 
The base-promoted ring closure in acyclic alkynyl ketones and alcohols occurs via the addition of oxygenbased nucleophilic group to the carbon-carbon triple bond. Agreement with Baldwin rules for ring formation cyclization can proceed along two pathways (5-exo-dig or 6-endo-dig cyclization) to give either 2-alkylfurans or $4 H$-pyrans, respectively (Figure 1)..$^{31,32}$

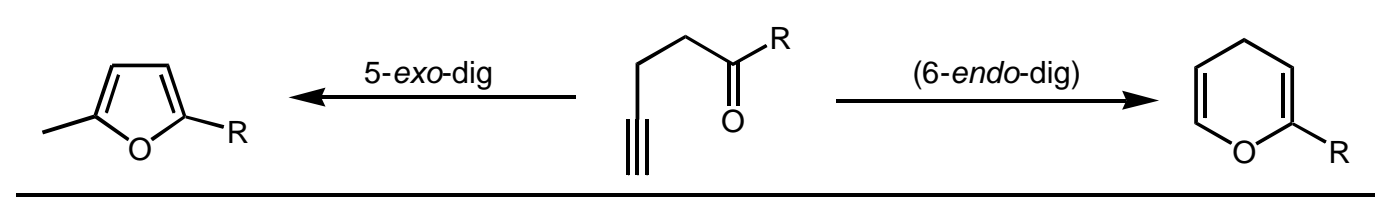

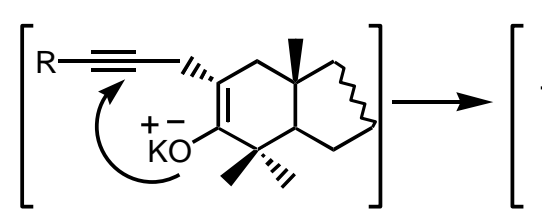

A

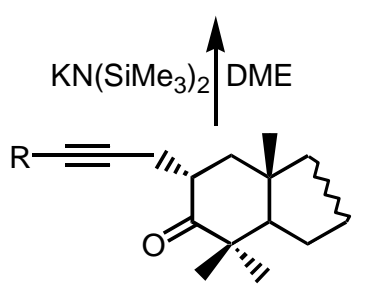

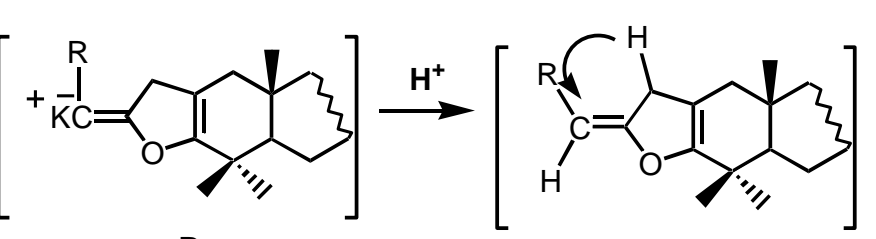

B

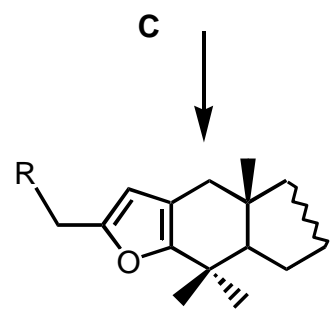

Figure 1. Assumed pathway to furan ring formation.

The KN(SiMe $)_{2}$-promoted cyclization of compounds 8-10, 14a-g, 17, and 19 proceeded with high regioselectivity as a 5-exo-dig cyclization according to the probable ${ }^{21,22}$ pathway shown in Figure 1. Apparently, elimination of the methine proton at the C-2 atom of ring A of triterpenoids 8-10, 14a-g, 17, and 19 is followed by 5-exo-dig attack by the nucleophilic enolate oxygen on the triple bond of intermediate $A$ to give intermediate $\mathbf{B}$. The protodemetalation during hydrolysis of intermediate $\mathbf{B}$ affords an unstable alkylidene dihydrofuran intermediate $\mathbf{C}$, which undergoes a rapid isomerization to furan-fused triterpenoid.

\section{Conclusions}

We have developed an efficient procedure to synthesize new furan-fused lupane-, ursane, and oleane-type pentacyclic triterpenoids. The synthesis is based on $\mathrm{KN}\left(\mathrm{SiMe}_{3}\right)_{2-}$ or $\mathrm{BuOK}^{\mathrm{t}}$-promoted cycloisomerization of accessible 2-alkynyl-3-oxotriterpene acid derivatives in dimethoxyethane. Future research will address transition metal complex-catalyzed heterocyclization of acetylene derivatives of triterpene acids.

\section{Experimental Section}

General. IR spectra were recorded on a Specord IR-75 spectrometer (thin films or solutions in $\mathrm{CHCl}_{3}$ ). ${ }^{1} \mathrm{H}$ and ${ }^{13} \mathrm{C}$ NMR spectra were recorded on a Bruker Avance-500 instrument $\left(500.13\left({ }^{1} \mathrm{H}\right)\right.$ and $\left.125.78 \mathrm{MHz}\left({ }^{13} \mathrm{C}\right)\right)$ or on a Bruker Avance-400 instrument $\left(400.13\left({ }^{1} \mathrm{H}\right)\right.$ and $\left.100.62 \mathrm{MHz}\left({ }^{13} \mathrm{C}\right)\right)$ in $\mathrm{CDCl}_{3}$ with $\mathrm{Me}_{4} \mathrm{Si}$ as the internal standard. Mass spectra of new compounds were recorded on a Bruker-Autoflex III spectrometer (MALDI TOF, 
positive ion mode, sinapic acid as the matrices) or on an LCMS-2010 EV (Shimadzu) spectrometer of the UfIC RAS Center for Collective Use "Chemistry". The measurements were performed on the positive and negative ions. Solution of compounds in acetonitrile: $\mathrm{H}_{2} \mathrm{O}(95: 5)$ were injected, using a syringe the flow rate $0.1 \mathrm{ml} \mathrm{min}^{-1}$. The carrier gas was nitrogen $(2.0 \mathrm{~L} \mathrm{~min}-1)$, the interface temperature was $250{ }^{\circ} \mathrm{C}$. Optical rotation was determined on a Perkin-Elmer-141 polarimeter. Specific rotation $[\alpha]_{D}$ is expressed in $(\mathrm{deg} \mathrm{mL}) /(\mathrm{g} \mathrm{dm})$; the concentration of the solution $c$ is expressed in $\mathrm{g} / 100 \mathrm{~mL}$. Elemental analysis was carried out on a Carlo Erba 1106 analyzer. TLC was carried out on Sorbfil plates (Sorbpolimer, Krasnodar, Russia) in hexane-EtOAc (from 30:1 to 1:1); spots were visualized with anisaldehyde. Silica gel L (KSKG grade, 50-160 $\mu \mathrm{m}$ ) was employed for column chromatography. The starting compounds betulin, betulinic acid, ursolic acid and reagents: $\mathrm{BEt}_{3}(95 \%)$, $\mathrm{KN}\left(\mathrm{SiMe}_{3}\right)_{2}$ (1 M solution in THF), propargyl bromide, Lil, Cul, $\mathrm{PdCl}_{2}\left(\mathrm{PPh}_{3}\right)_{2}$, aryl iodide $\left(\mathrm{C}_{6} \mathrm{H}_{5} \mathrm{I}, \mathrm{IC}_{6} \mathrm{H}_{4} \mathrm{Br}_{2}, \mathrm{IC}_{6} \mathrm{H}_{4} \mathrm{Cl}\right.$, $\mathrm{IC}_{6} \mathrm{H}_{4} \mathrm{~F}, \quad \mathrm{IC}_{6} \mathrm{H}_{4} \mathrm{NO}_{2}, \quad \mathrm{IC}_{6} \mathrm{H}_{2}\left(\mathrm{OCH}_{3}\right)_{3}, \quad \mathrm{IC}_{6} \mathrm{H}_{4} \mathrm{CH}_{3}, \mathrm{Et}_{3} \mathrm{~N}$, DMF, DME (dimethoxyethane), (Aldrich). Betulonic and betulinic acids were obtained from betulin according to known procedures. ${ }^{33}$

\section{General procedure for the synthesis of [3,2-b]furano-fused triterpenoids 11a-13a}

Starting triterpenoids 8-10 were prepared as previously reported. ${ }^{28}$ A $1 \mathrm{M}$ solution of $\mathrm{KN}\left(\mathrm{SiMe}_{3}\right)_{2}(0.51 \mathrm{~mL}$, $0.51 \mathrm{mmol})$ in THF was added to a solution of triterpenoids 8-10 $(0.39 \mathrm{mmol})$ in DME $(6 \mathrm{~mL})$. The reaction mixture was stirred at room temperature under an argon atmosphere. The completion of reaction was monitored by TLC analysis. After 30 min reaction mixture was neutralized with $5 \% \mathrm{HCl}(\mathrm{aq})$. The product was extracted with EtOAc $(3 \times 10 \mathrm{~mL})$. The combined extracts were dried with $\mathrm{MgSO}_{4}$ and concentrated. The residue was purified by column chromatography on $\mathrm{SiO}_{2}$ with hexane/EtOAc (30:1) as an eluent to give the appropriate compound 11a-13a.

Methyl 5'-methylfurano[3,2-b]lup-20(29)-en-28-oate (11a). Colorless crystals (58\%). mp 71-73 ${ }^{\circ} \mathrm{C} .[\alpha]_{\mathrm{D}}^{21}+29.7^{\circ}$ (c $0.53, \mathrm{CHCl}_{3}$ ). ${ }^{1} \mathrm{H}$ NMR $\left(400 \mathrm{MHz} \mathrm{CDCl}_{3}\right): \delta_{\mathrm{H}} 5.68(\mathrm{~s}, 1 \mathrm{H}, \mathrm{H}-4$ '), 4.78, 4.64 (both br s, 2H, H-29), 3.70 (s, 3H, OMe), 3.05 (m, 1H, H-19), 2.35 (d, 1H, J $\left.15 \mathrm{~Hz}, \mathrm{H}^{\mathrm{a}}-1\right), 2.26$ (s, 3H, Me-5'), 2.29-1.34 (m, 21H, CH, CH pentacyclic skeleton and $\left.1 \mathrm{H}, \mathrm{H}^{\mathrm{b}}-1\right), 1.72(\mathrm{~s}, 3 \mathrm{H}, \mathrm{H}-30), 1.22,1.11,1.01,0.99,0.86$ (all s, 3H each, H-23-H27). ${ }^{13} \mathrm{C}$ NMR $\left(100 \mathrm{MHz}_{\mathrm{C}} \mathrm{CDCl}_{3}\right): \delta_{\mathrm{c}} 176.67,154.45,150.57,149.58,113.68,109.62,106.78,56.60,53.62,51.27$, 49.45, 49.22, 46.97, 42.41, 40.72, 38.64, 38.57, 38.40, 36.97, 34.60, 33.51, 32.15, 30.62, 29.79, 29.16, 25.63, 21.39, 21.39, 19.40, 18.98, 16.30, 15.70, 14.72, 13.72. Anal. Calcd for $\mathrm{C}_{34} \mathrm{H}_{50} \mathrm{O}_{3}: \mathrm{C}, 80.58 ; \mathrm{H}, 9.94$. Found: $\mathrm{C}$, 80.61; $\mathrm{H}, 9.91 . \mathrm{MS}(\mathrm{APCl}): \mathrm{m} / \mathrm{z}[\mathrm{M}+\mathrm{H}]^{+}$, found 507. $\left[\mathrm{C}_{34} \mathrm{H}_{50} \mathrm{O}_{3}\right]^{+}$requires 506.38 .

Methyl 5'-methylfurano[3,2-b]urs-12-en-28-oate (12a). Colorless crystals (56\%). mp 76-78 ${ }^{\circ} \mathrm{C} .[\alpha]_{\mathrm{D}}^{19}+80.7^{\circ}(c$ 0.60, $\left.\mathrm{CHCl}_{3}\right) .{ }^{1} \mathrm{H}$ NMR $\left(500 \mathrm{MHz}, \mathrm{CDCl}_{3}\right): \delta_{\mathrm{H}} 5.69\left(\mathrm{~s}, 1 \mathrm{H}, \mathrm{H}-4{ }^{\prime}\right), 5.33(\mathrm{~m}, 1 \mathrm{H}, \mathrm{H}-12), 3.64(\mathrm{~s}, 3 \mathrm{H}, \mathrm{OMe}), 2.33(\mathrm{~d}, 1 \mathrm{H}$, $\left.J 15 \mathrm{~Hz}, \mathrm{H}^{\mathrm{a}}-1\right), 2.28(\mathrm{~m}, 1 \mathrm{H}, \mathrm{H}-18), 2.26\left(\mathrm{~s}, 3 \mathrm{H}, \mathrm{Me}-5^{\prime}\right), 2.05-1.03\left(\mathrm{~m}, 19 \mathrm{H}, \mathrm{CH}, \mathrm{CH}_{2}\right.$ in pentacyclic skeleton and $\left.1 \mathrm{H}, \mathrm{H}^{\mathrm{b}}-1\right), 1.23,1.13,1.12,0.95,0.82$ (all s, 3H each, $\left.\mathrm{H}-23-\mathrm{H}-27\right), 0.96(\mathrm{~d}, 3 \mathrm{H}, J 5 \mathrm{~Hz}, \mathrm{H}-30), 0.88(\mathrm{~d}, 3 \mathrm{H}, J 5 \mathrm{~Hz}, \mathrm{H}-$ 30). ${ }^{13} \mathrm{C}$ NMR $\left(125 \mathrm{MHz}, \mathrm{CDCl}_{3}\right): \delta_{c} 178.10,154.41,149.64,138.04,125.73,113.62,106.65,53.56,53.04,51.47$, 49.11, 48.19, 42.14, 39.58, 39.14, 38.89, 38.37, 38.29, 36.66, 34.48, 32.42, 30.70, 29.36, 28.08, 24.29, 23.49, 23.36, 21.50, 21.18, 19.02, 17.04, 16.80, 15.67, 13.72. Anal. Calcd for $\mathrm{C}_{34} \mathrm{H}_{50} \mathrm{O}_{3}$ : C, 80.58; $\mathrm{H}, 9.94$. Found: C, 80.47; $\mathrm{H}, 9.85 . \mathrm{MS}(\mathrm{APCl}): \mathrm{m} / \mathrm{z}[\mathrm{M}+\mathrm{H}]^{+}$, found 507. $\left[\mathrm{C}_{34} \mathrm{H}_{50} \mathrm{O}_{3}\right]^{+}$requires 506.38.

Methyl 5'-methylfurano[3,2-b]olean-12-en-28-oate (13a). Colorless crystals (54\%). mp $80-82{ }^{\circ} \mathrm{C} .[\alpha]_{\mathrm{D}}^{21}$

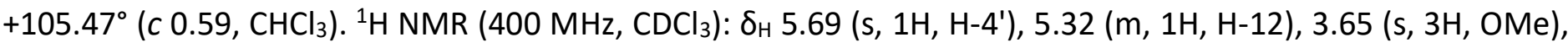
$2.91(\mathrm{~m}, 1 \mathrm{H}, \mathrm{H}-18), 2.30\left(\mathrm{~d}, 1 \mathrm{H}, J 15 \mathrm{~Hz}, \mathrm{H}^{\mathrm{a}}-1\right), 2.26\left(\mathrm{~s}, 3 \mathrm{H}, \mathrm{Me}-5^{\prime}\right), 2.02-1.22\left(\mathrm{~m}, 19 \mathrm{H}, \mathrm{CH}, \mathrm{CH}_{2}\right.$ in pentacyclic skeleton and $\left.1 \mathrm{H}, \mathrm{H}^{\mathrm{b}}-1\right), 1.23,1.17,1.13,0.94,0.81$ (all s, 3H each, $\left.\mathrm{H}-23-\mathrm{H}-27\right), 0.93(\mathrm{~d}, 3 \mathrm{H}, J 5 \mathrm{~Hz}, \mathrm{H}-30), 0.96$ (d, $3 \mathrm{H}, J 5 \mathrm{~Hz}, \mathrm{H}-30,) .{ }^{13} \mathrm{C} N M R\left(100 \mathrm{MHz}, \mathrm{CDCl}_{3}\right): \delta_{c} 178.31,154.45,149.63,143.63,122.52,113.57,106.66$, 53.53, 51.54, 46.80, 46.19, 45.90, 41.80, 41.43, 39.38, 38.37, 38.11, 34.48, 33.90, 33.13, 32.39, 32.10, 30.71, 
29.33, 27.77, 25.77, 23.63, 23.40, 23.14, 21.52, 19.05, 16.61, 15.50, 13.72. Anal. Calcd for $\mathrm{C}_{34} \mathrm{H}_{50} \mathrm{O}_{3}: \mathrm{C}, 80.58$; $\mathrm{H}, 9.80$. Found: $\mathrm{C}, 80.49 ; \mathrm{H}, 9.93 . \mathrm{m} / \mathrm{z}[\mathrm{M}+\mathrm{H}]^{+}$, found 507. $\left[\mathrm{C}_{34} \mathrm{H}_{50} \mathrm{O}_{3}\right]^{+}$requires 506.38 .

General procedure for the synthesis of 2 $\alpha$-arylpropynyl triterpenoids derivatives $14 \mathrm{a}-\mathrm{g}, 17$ and 19 via Sonogashira coupling reaction. An oven-dried flask equipped with a magnetic stirring was flushed with argon. Then to a mixture of corresponding triterpenoids $(0.3 \mathrm{mmol})$ and an aryl iodide $(0.25 \mathrm{mmol})$ were dissolved in DMF (4.5 mL), Et ${ }_{3} \mathrm{~N}\left(0.34 \mathrm{~mL}, 2.47 \mathrm{mmol}\right.$ ) was added. Then Cul $(4.6 \mathrm{mg}, 0.03 \mathrm{mmol})$ and $\mathrm{PdCl}_{2}\left(\mathrm{PPh}_{3}\right)_{2}(8.6 \mathrm{mg}$, $0.01 \mathrm{mmol}$ ) were added to the mixture simultaneously and the resulting mixture was stirred at room temperature for 1-3 hours. The completion of reaction was monitored by TLC analysis. The reaction was quenched by addition of water and extracted with EtOAc $(3 \times 10 \mathrm{~mL})$. The combined organic extracts were dried with $\mathrm{MgSO}_{4}$ and concentrated under reduced pressure. The residue was purified by column chromatography on $\mathrm{SiO}_{2}$ with hexane/EtOAc (30:1) as an eluent to give a final compound: 14a-g, 17 and 19.

Methyl 2 $\alpha$-phenylpropynyl-3-oxolup-20(29)-en-28-oate (14a). Colorless crystals (69\%). mp 74-76 ${ }^{\circ} \mathrm{C} .[\alpha]_{\mathrm{D}}^{24}$ $67.6^{\circ}$ (c $0.68, \mathrm{CHCl}_{3}$ ). ${ }^{1} \mathrm{H} \mathrm{NMR}\left(500 \mathrm{MHz}, \mathrm{CDCl}_{3}\right): \delta_{\mathrm{H}} 7.40-7.28(\mathrm{~m}, 5 \mathrm{H}$, arom), 4.75, 4.61 (both br s, 2H, H-29), 3.69 (s, 3H, OMe), 3.03 (m, 1H, H-19), 2.94 (m, 1H, H-2), 2.44 (dd, 1H, J 15.0, 5.0 Hz, Ha -1 ), 2.87 and $2.27(1 \mathrm{H}$ each, dd, J 15.0, $5.0 \mathrm{~Hz}, \mathrm{CH}_{2}$ in propargyl), $1.69(\mathrm{~s}, 3 \mathrm{H}, \mathrm{H}-30), 2.28-1.12\left(\mathrm{~m}, 21 \mathrm{H}, \mathrm{CH}, \mathrm{CH}_{2}\right.$ in pentacyclic skeleton and $\left.1 \mathrm{H}, \mathrm{H}^{\mathrm{b}}-1\right), 1.16,1.10,1.08,1.01,0.97$ (all s, 3H each, $\left.\mathrm{H}-23-\mathrm{H}-27\right) .{ }^{13} \mathrm{C} \mathrm{NMR}\left(125 \mathrm{MHz}, \mathrm{CDCl}_{3}\right): \delta_{\mathrm{c}} 215.98$, $176.61,150.42,131.62,128.18,127.58,123.92,109.73,88.65,81.71,57.32,56.48,51.29,50.14,49.43,48.30$, $46.98,46.79,42.52,41.76,40.79,38.22,37.47,36.95,34.08,32.15,30.56,29.63,25.43,25.11,21.71,21.19$, 20.53, 19.33, 19.33, 16.13, 16.13, 14.65. Anal. Calcd for $\mathrm{C}_{40} \mathrm{H}_{54} \mathrm{O}_{3}: \mathrm{C}, 82.43 ; \mathrm{H}, 9.34$. Found: $\mathrm{C}, 82.39 ; \mathrm{H}, 9.12 \%$.

Methyl 2 $\alpha$-(4-bromophenylpropynyl)-3-oxolup-20(29)en-28-oate (14b). Colorless crystals (71\%). $\mathrm{mp} 88-90{ }^{\circ} \mathrm{C}$. $[\alpha]_{\mathrm{D}}^{21}-54.64^{\circ}\left(\mathrm{c} 0.64, \mathrm{CHCl}_{3}\right) .{ }^{1} \mathrm{H}$ NMR $\left(400 \mathrm{MHz}, \mathrm{CDCl}_{3}\right): \delta_{\mathrm{H}} 7.42(\mathrm{~d}, 2 \mathrm{H}, \mathrm{J} 8 \mathrm{~Hz}$ arom), $7.25(\mathrm{~d}, 2 \mathrm{H}, \mathrm{J} 8 \mathrm{~Hz}, \mathrm{arom})$, 4.75, 4.61 (both br s, 2H, H-29), 3.69 (s, 3H, OMe), 3.03 (m, 1H, H-19), 2.95 (m, 1H, H-2), 2.40 (dd, 1H, J 15.0, $5.0 \mathrm{~Hz}, \mathrm{H}^{\mathrm{a}}-1$ ), 2.85 and 2.37 (1H each, dd, J 15.0, $5.0 \mathrm{~Hz}, \mathrm{CH}_{2}$ in propargyl), 1.69 (s, 3H, H-30), $2.28-1.12$ (m, $21 \mathrm{H}, \mathrm{CH}, \mathrm{CH}_{2}$ in pentacyclic skeleton and $1 \mathrm{H}, \mathrm{H}^{\mathrm{b}}-1$ ), 1.15, 1.09, 1.08, 1.00, 0.97 (all s, 3H each, $\mathrm{H}-23-\mathrm{H}-27$ ). ${ }^{13} \mathrm{C}$ NMR $\left(100 \mathrm{MHz}_{\mathrm{CDCl}}\right.$ ): $\delta_{c} 215.81,176.60,150.45,133.09,131.40,122.87,121.68,109.69,90.00,80.68,57.32$, 56.48, 51.29, 50.14, 49.42, 48.31, 46.96, 46.83, 42.52, 41.65, 40.79, 38.21, 37.48, 36.93, 34.07, 32.13, 30.56, 29.62, 25.42, 25.08, 21.68, 21.19, 20.56, 19.34, 19.34, 16.13, 16.13, 14.64. Anal. Calcd for $\mathrm{C}_{40} \mathrm{H}_{53} \mathrm{BrO}_{3}: \mathrm{C}_{7}$ 72.63; $H, 7.98$. Found: $C, 72.63 ; H, 7.98 \%$.

Methyl $2 \alpha$-(4-chlorophenylpropynyl)-3-oxolup-20(29)en-28-oate (14c). Colorless crystals (70\%). mp 64-66 ${ }^{\circ} \mathrm{C}$.

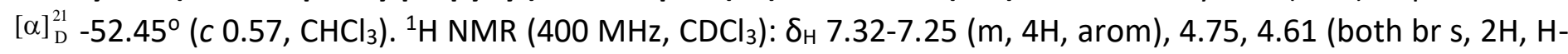
29), 3.69 (s, 3H, OMe), 3.03 (m, 1H, H-19), 2.93 (m, 1H, H-2), 2.44 (dd, 1H, J 15.0, 5.0 Hz, Ha -1 ), 2.85 and 2.37 (1H each, dd, J 15.0, $5.0 \mathrm{~Hz}, \mathrm{CH}_{2}$ in propargyl), $1.69(\mathrm{~s}, 3 \mathrm{H}, \mathrm{H}-30), 2.27-1.12\left(\mathrm{~m}, 21 \mathrm{H}, \mathrm{CH}_{2} \mathrm{CH}_{2}\right.$ in pentacyclic skeleton and $\left.1 \mathrm{H}, \mathrm{H}^{\mathrm{b}}-1\right), 1.16,1.09,1.08,1.00,0.97$ (all s, 3H each, $\left.\mathrm{H}(23)-\mathrm{H}(27)\right) .{ }^{13} \mathrm{C} \mathrm{NMR}\left(100 \mathrm{MHz} \mathrm{CDCl}_{3}\right): \delta_{\mathrm{c}}$ 215.83, 176.60, 150.45, 133.52, 132.84, 128.47, 122.40, 109.69, 89.77, 80.62, 57.32, 56.48, 51.28, 50.14, $49.42,48.31$,46.96, 46.84, 42.52, 41.67, 40.79, 38.21, 37.48, 36.93, 34.07, 32.13, 30.56, 29.62, 25.43, 25.08, 21.68, 21.19, 20.53, 19.33, 19.33, 16.12, 16.12, 14.63. Anal. Calcd for $\mathrm{C}_{40} \mathrm{H}_{53} \mathrm{ClO}_{3}: \mathrm{C}, 77.83 ; \mathrm{H}, 8.65$. Found: C, $77.91 ; \mathrm{H}, 8.72 \%$.

Methyl 2 $\alpha$-(4-fluorophenylpropynyl)-3-oxolup-20(29)en-28-oate (14d). Colorless crystals (73\%). $\mathrm{mp} 70-73{ }^{\circ} \mathrm{C}$. $[\alpha]_{\mathrm{D}}^{20}-64^{\circ}$ (c 1.05, $\mathrm{CHCl}_{3}$ ). 1H NMR (400 MHz, $\left.\mathrm{CDCl}_{3}\right): \delta_{\mathrm{H}} 7.38-7.28(\mathrm{~m}, 2 \mathrm{H}$, arom), 7.01-6.96 (m, 2H, arom), 4.75, 4.61 (both br s, 2H, H-29), 3.69 (s, 3H, OMe), 3.02 (m, 1H, H-19), $2.92(m, 1 H, H-2), 2.40\left(m, 1 H, H^{a}-1\right)$, 2.85 and 2.37 ( $1 \mathrm{H}$ each, dd, $J 15.0,5.0 \mathrm{~Hz}, \mathrm{CH}_{2}$ in propargyl), $1.69(\mathrm{~s}, 3 \mathrm{H}, \mathrm{H}-30), 2.27-1.12\left(\mathrm{~m}, 21 \mathrm{H}, \mathrm{CH}, \mathrm{CH}_{2}\right.$ in pentacyclic skeleton and $1 \mathrm{H}, \mathrm{H}^{\mathrm{b}}-1$ ), 1.16, 1.09, 1.08, 1.00, 0.97 (all s, 3H each, $\mathrm{H}(23)-\mathrm{H}(27)$ ). ${ }^{13} \mathrm{C} \mathrm{NMR} \mathrm{(100}$ $\mathrm{MHz}_{\mathrm{CDCl}}$ ): $\delta_{\mathrm{C}} 215.90,176.60,163.67,161.01,150.46,133.43,133.35,119.94,115.48,115.26,109.68,88.27$, $80.59,51.29,57.32,56.48,50.14,49.41,48.31,46.96,46.83,42.52,41.71,40.79,38.21,37.48,36.93,34.07$, 
$32.13,30.56,29.62,25.43,25.08,21.69,21.19,20.46,19.32,19.32,16.12,16.12$, 14.63. Anal. Calcd for $\mathrm{C}_{40} \mathrm{H}_{53} \mathrm{FO}_{3}: \mathrm{C}, 79.96 ; \mathrm{H}, 8.89$. Found: $\mathrm{C}, 79.89 ; \mathrm{H}, 8.90 \%$.

Methyl 2 $\alpha$-(2-methylphenylpropynyl)-3-oxolup-20(29)en-28-oate (14e). Colorless crystals (72\%). mp 60-63 ${ }^{\circ} \mathrm{C} .[\alpha]_{\mathrm{D}}^{24}-42.9^{\circ}\left(\mathrm{c} 0.57, \mathrm{CHCl}_{3}\right) .{ }^{1} \mathrm{H} \mathrm{NMR}\left(400 \mathrm{MHz}, \mathrm{CDCl}_{3}\right): \delta_{\mathrm{H}} 7.12-7.38(\mathrm{~m}, 4 \mathrm{H}$, arom), 4.75, 4.61 (both br s, $2 \mathrm{H}$, $\mathrm{H}-29), 3.70$ (s, 3H, OMe), $3.02(\mathrm{~m}, 1 \mathrm{H}, \mathrm{H}-19), 3.00(\mathrm{~m}, 1 \mathrm{H}, \mathrm{H}-2), 2.48\left(\mathrm{dd}, 1 \mathrm{H}, J\right.$ 15.0, $\left.5.0 \mathrm{~Hz}, \mathrm{H}^{\mathrm{a}}-1\right), 2.42$ (s, 3H, Me- $\left.\mathrm{C}_{6} \mathrm{H}_{4}\right), 2.80$ and 2.25 (1H each, dd, J 15.0, $5.0 \mathrm{~Hz}, \mathrm{CH}_{2}$ in propargyl), $1.70(\mathrm{~s}, 3 \mathrm{H}, \mathrm{H}-30), 2.28-1.12(\mathrm{~m}, 21 \mathrm{H}$, $\mathrm{CH}, \mathrm{CH}_{2}$ in pentacyclic skeleton and $\left.1 \mathrm{H}, \mathrm{H}^{\mathrm{b}}-1\right), 1.17,1.10,1.09,1.01,0.98\left(3 \mathrm{H}\right.$ each, all s, H-23-H-27). ${ }^{13} \mathrm{C} \mathrm{NMR}$ $\left(100 \mathrm{MHz}_{1} \mathrm{CDCl}_{3}\right): \delta_{\mathrm{C}} 215.94,176.63,150.39,139.95,131.79,129.28,125.43,123.69,121.68,109.72,92.69$, 80.61, 57.39, 56.50, 51.29, 50.19, 49.42, 48.34, 46.97, 46.80, 42.52, 41.88, 40.79, 38.22, 37.49, 36.95, 34.10, $32.16,30.57,29.63,25.41,25.05,21.71,21.15,20.85,20.64,19.33,19.33,16.10,16.10,14.63$. Anal. Calcd for $\mathrm{C}_{41} \mathrm{H}_{56} \mathrm{O}_{3}: \mathrm{C}, 82.50 ; \mathrm{H}, 9.46$. Found: $\mathrm{C}, 82.61 ; \mathrm{H}, 9.41 \%$.

Methyl 2 $\alpha$-(3,4,5-trimethoxyphenylpropynyl)-3-oxolup-20(29)en-28-oate (14f). Colorless crystals (75\%). mp $92-95^{\circ} \mathrm{C} .[\alpha]_{\mathrm{D}}^{20}-59.7^{\circ}$ (c 0.77, $\mathrm{CHCl}_{3}$ ). $1 \mathrm{H} \mathrm{NMR}\left(500 \mathrm{MHz}, \mathrm{CDCl}_{3}\right): \delta_{\mathrm{H}} 6.65$ (s, 2H, arom), 4.73, 4.59 (both br s, $2 \mathrm{H}$, $\mathrm{H}-29$ ), 3.84 (s, 9H, Ph(OMe) 3 ), 3.68 (s, 3H, OMe), 3.01 (m, 1H, H-19), 2.92 (m, 1H, H-2), 2.45 (dd, 1H, J 15.0, 5.0 $\mathrm{Hz}, \mathrm{H}^{\mathrm{a}}-1$ ), 2.80 and 2.39 (1H each, dd, $J 15.0,5.0 \mathrm{~Hz}, \mathrm{CH}_{2}$ in propargyl), $1.68(\mathrm{~s}, 3 \mathrm{H}, \mathrm{H}-30), 2.27-1.17(\mathrm{~m}, 21 \mathrm{H}$, $\mathrm{CH}, \mathrm{CH}_{2}$ in pentacyclic skeleton and $\left.1 \mathrm{H}, \mathrm{H}^{\mathrm{b}}-1\right), 1.15,1.09,1.07,1.00,0.96$ (3H each, all s, H-23-H-27). ${ }^{13} \mathrm{C} \mathrm{NMR}$ $\left(125 \mathrm{MHz}, \mathrm{CDCl}_{3}\right): \delta_{\mathrm{C}} 215.92,176.59,152.96,150.50,138.31,118.96,109.66,108.77,87.76,81.86,51.30$, 60.93, 57.27, 56.46, 56.06, 50.13, 49.39, 48.31, 46.97, 46.76, 42.51, 41.60, 40.77, 38.21, 37.42, 36.92, 34.06, $32.11,30.51,29.61,25.44,25.11,21.71,21.22,20.45,19.30,19.30,16.13,16.11,14.62$. Anal. Calcd for $\mathrm{C}_{43} \mathrm{H}_{60} \mathrm{O}_{6}$ : C, 76.75; $\mathrm{H}, 8.99$. Found: $\mathrm{C}, 76.82 ; \mathrm{H}, 9.05 \%$.

Methyl 2 $\alpha$-(4-nitrophenylpropynyl)-3-oxolup-20(29)en-28-oate (14g). Colorless crystals (71\%). $\mathrm{mp} 70-72{ }^{\circ} \mathrm{C}$. $[\alpha]_{\mathrm{D}}^{24}-64.4^{\circ}\left(\mathrm{c} 0.65, \mathrm{CHCl}_{3}\right) .{ }^{1} \mathrm{H} \mathrm{NMR}\left(500 \mathrm{MHz}, \mathrm{CDCl}_{3}\right): \delta_{\mathrm{H}} 8.15(\mathrm{~d}, 2 \mathrm{H}, J 8 \mathrm{~Hz}$, arom), $7.52(\mathrm{~d}, 2 \mathrm{H}, J 8 \mathrm{~Hz}, \mathrm{arom})$, 4.75, 4.61 (both br s, 2H, H-29), 3.69 (s, 3H, OMe), $3.02(\mathrm{~m}, 1 \mathrm{H}, \mathrm{H}-19), 2.97(\mathrm{~m}, 1 \mathrm{H}, \mathrm{H}-2), 2.88$ and $2.41(1 \mathrm{H}$ each, dd, J 15.0, $5.0 \mathrm{~Hz}, \mathrm{CH}_{2}$ in propargyl), 2.47 (dd, $\left.1 \mathrm{H}, J 15.0,5.0 \mathrm{~Hz}, \mathrm{H}^{\mathrm{a}}-1\right), 1.69(\mathrm{~s}, 3 \mathrm{H}, \mathrm{H}-30), 2.28-1.10(\mathrm{~m}$, $21 \mathrm{H}, \mathrm{CH}, \mathrm{CH}_{2}$ in pentacyclic skeleton and $1 \mathrm{H}, \mathrm{H}^{\mathrm{b}}-1$ ), 1.17, 1.10, 1.09, 1.01, 0.97 (all s, 3H each, $\mathrm{H}(23)-\mathrm{H}(27)$ ). ${ }^{13} \mathrm{C}$ NMR $\left(125 \mathrm{MHz}_{2} \mathrm{CDCl}_{3}\right): \delta_{\mathrm{c}} 215.53,176.61,150.51,146.67,132.35,130.94,123.94,109.66,95.02,80.30,57.36$, $56.48,51.31,50.18,49.40,48.39,46.95,46.95,42.53,41.53,40.80,38.19,37.54,36.93,34.06,32.12,30.55$, $29.62,25.42,25.04,21.66,21.20,20.72,19.30,19.30,16.17,16.17,14.63$. Anal. Calcd for $\mathrm{C}_{40} \mathrm{H}_{53} \mathrm{NO}_{5}: \mathrm{C}, 76.52$; $\mathrm{H}$, 8.51. Found: $\mathrm{C}, 76.48 ; \mathrm{H}, 8.56 \%$.

Methyl 2 $\alpha$-phenylpropynyl-3-oxours-12en-28-oate (17). Colorless crystals (75\%). mp 78-80 ${ }^{\circ} \mathrm{C} .[\alpha]_{\mathrm{D}}^{19}-40.9^{\circ}(c$ 0.59, $\mathrm{CHCl}_{3}$ ). ${ }^{1} \mathrm{H} \mathrm{NMR}\left(500 \mathrm{MHz}, \mathrm{CDCl}_{3}\right): \delta_{\mathrm{H}} 7.41-7.30(\mathrm{~m}, 5 \mathrm{H}, \mathrm{arom}), 5.29(\mathrm{~m}, 1 \mathrm{H}, \mathrm{H}-12), 3.63(\mathrm{~s}, 3 \mathrm{H}, \mathrm{OMe}), 2.96$ $(\mathrm{m}, 1 \mathrm{H}, \mathrm{H}-2), 2.44\left(\mathrm{~d}, 1 \mathrm{H}, J 15.0 \mathrm{~Hz}, \mathrm{H}^{\mathrm{a}}-1\right), 2.90$ and 2.30 (1H each, dd, J 15.0, $5.0 \mathrm{~Hz}, \mathrm{CH}_{2}$ in propargyl), 2.26 (m, $1 \mathrm{H}, \mathrm{H}-18), 2.06-1.03\left(\mathrm{~m}, 19 \mathrm{H}, \mathrm{CH}, \mathrm{CH}_{2}\right.$ in pentacyclic skeleton and $\left.1 \mathrm{H}, \mathrm{H}^{\mathrm{b}}-1\right), 1.26,1.12,1.11,1.09,0.84$ (all s, $3 \mathrm{H}$ each, H-23-H-27), 0.94 (d, J $6 \mathrm{~Hz}, 3 \mathrm{H}, \mathrm{H}-30), 0.86$ (d, J $6 \mathrm{~Hz}, 3 \mathrm{H}, \mathrm{H}-29) .{ }^{13} \mathrm{C} \mathrm{NMR}\left(125 \mathrm{MHz}, \mathrm{CDCl}_{3}\right): \delta_{\mathrm{C}} 215.91$, $178.02,138.41,131.60,128.21,127.63,125.20,123.90,88.66,81.78,57.18,52.86,51.48,48.26,48.08,47.06$, $46.47,42.11,41.72,39.59,39.02,38.86,37.18,36.61,32.71,30.63,28.02,25.36,24.19,23.61,23.52,22.00$, 21.18, 20.54, 19.38, 17.10, 17.10, 15.62. Anal. Calcd for $\mathrm{C}_{40} \mathrm{H}_{54} \mathrm{O}_{3}: \mathrm{C}, 82.43 ; \mathrm{H}, 9.34$. Found: $\mathrm{C}, 82.50 ; \mathrm{H}, 9.31 \%$.

Methyl $2 \alpha$-phenylpropynyl-3-oxoolean-12en-28-oate (19). Colorless crystals (81\%). mp 76-79 ${ }^{\circ} \mathrm{C}$. $[\alpha]_{\mathrm{D}}^{21}-14.52^{\circ}$ (c 0.79, $\left.\mathrm{CHCl}_{3}\right) .{ }^{1} \mathrm{H}$ NMR (400 MHz, $\left.\mathrm{CDCl}_{3}\right): \delta_{\mathrm{H}} 7.41-7.39(\mathrm{~m}, 2 \mathrm{H}$, arom), 7.31-7.29 (m, 3H, arom), $5.32(\mathrm{~m}, 1 \mathrm{H}, \mathrm{H}-$ 12), 3.65 (s, 3H, OMe), $2.94(\mathrm{~m}, 1 \mathrm{H}, \mathrm{H}-2), 2.87$ and 2.43 (1H each, dd, J 15.0, $5.0 \mathrm{~Hz}, \mathrm{CH}_{2}$ of propargyl, 2.36 (d, $1 \mathrm{H}, J$ 15.0, $\left.5.0 \mathrm{~Hz}, \mathrm{H}^{\mathrm{a}}-1\right), 2.01-1.16\left(\mathrm{~m}, 2 \mathrm{H}, \mathrm{CH}, \mathrm{CH}_{2}\right.$ in pentacyclic skeleton and $\left.1 \mathrm{H}, \mathrm{H}^{\mathrm{b}}-1\right), 1.25,1.14,1.12$, $1.11,0.82$ (all s, 3H each, H-23-H-27), 0.95 (s, 3H, H-29), $0.91(\mathrm{~s}, 3 \mathrm{H}, \mathrm{H}-30) .{ }^{13} \mathrm{C} \mathrm{NMR}\left(125 \mathrm{MHz}, \mathrm{CDCl}_{3}\right): \delta_{\mathrm{c}}$ $215.89,178.25,143.97,131.59,128.20,127.61,123.92,122.03,88.62,81.78,57.17,51.55,48.27,47.17$, $46.72,46.30,45.84,41.76,41.62,41.33,39.38,37.24,33.85,33.11,32.42,32.35,30.69,27.70,25.92,25.33$, 
23.64, 23.59, 23.05, 21.96, 20.50, 19.37, 17.03, 15.47. Anal. Calcd for $\mathrm{C}_{40} \mathrm{H}_{54} \mathrm{O}_{3}$ : C, 82.43; $\mathrm{H}, 9.34$. Found: C, 82.39; H, $9.27 \%$.

Synthesis of [3,2-b]furan-fused triterpenoids $15 a-g, 18 a$ and $20 a$. Synthesis of [3,2-b]furan fused triterpenoids 15a-g, 18a and 20a was carried out as described above for preparation of furans 11a-13a. The completion of reaction (in 10-12 $\mathrm{min}$ ) was monitored by TLC analysis.

Methyl 5'-benzylfurano[3,2-b]lup-20(29)-en-28-oate (15a). Colorless crystals (73\%). mp 76-79 ${ }^{\circ} \mathrm{C} .[\alpha]_{\mathrm{D}}^{21}+24.0^{\circ}$ (c 0.66, $\mathrm{CHCl}_{3}$ ). ${ }^{1} \mathrm{H}$ NMR $\left(400 \mathrm{MHz} \mathrm{CDCl}_{3}\right): \delta_{\mathrm{H}} 7.34-7.24(\mathrm{~m}, 5 \mathrm{H}$, arom), $5.64(\mathrm{~s}, 1 \mathrm{H}, \mathrm{H}-4$ '), 4.78, 4.64 (both br s, $2 \mathrm{H}, \mathrm{H}-29), 3.94$ (s, 2H, CH $\mathrm{CH}^{-}$) , 3.70 (s, 3H, OMe), 3.05 (m, 1H, H-19), 2.35 (d, 1H, J $\left.15.0 \mathrm{~Hz}, \mathrm{H}^{\mathrm{a}}-1\right), 2.28-1.27$ (m, $21 \mathrm{H}, \mathrm{CH}, \mathrm{CH}_{2}$ in pentacyclic skeleton and $\left.1 \mathrm{H}, \mathrm{H}^{\mathrm{b}}-1\right), 1.72(\mathrm{~s}, 3 \mathrm{H}, \mathrm{H}-30), 1.22,1.12,1.01,0.99,0.86$ (all s, 3H each, $\mathrm{H}-23-\mathrm{H}-27) .{ }^{13} \mathrm{C} N M R\left(125 \mathrm{MHz}, \mathrm{CDCl}_{3}\right): \delta_{\mathrm{c}} 176.68,155.11,152.20,150.58,138.74,128.76,128.35$, $126.22,113.69,109.62,107.59,56.61,53.59,51.28,49.46,49.20,46.98,42.42,40.73,38.60,38.60,38.40$, 36.97, 34.74, 34.66, 33.50, 32.15, 30.63, 29.13, 29.13, 25.63, 21.39, 21.39, 19.41, 18.97, 16.34, $15.70,14.71$. Anal. Calcd for $\mathrm{C}_{40} \mathrm{H}_{54} \mathrm{O}_{3}: \mathrm{C}, 82.43 ; \mathrm{H}, 9.34$. Found: $\mathrm{C}, 82.51 ; \mathrm{H}, 9.29$. MS (APCl): $\mathrm{m} / z[\mathrm{M}+\mathrm{H}]^{+}$, found 583 . $\left[\mathrm{C}_{40} \mathrm{H}_{54} \mathrm{O}_{3}\right]^{+}$requires 582.41 .

Methyl 5'-(4-bromobenzyl)furano[3,2-b]lup-20(29)-en-28-oate (15b). Colorless crystals (71\%). mp 96-98 ${ }^{\circ} \mathrm{C}$. $[\alpha]_{\mathrm{D}}^{21}+20.49^{\circ}\left(c 0.37, \mathrm{CHCl}_{3}\right) .{ }^{1} \mathrm{H} \mathrm{NMR}\left(400 \mathrm{MHz}, \mathrm{CDCl}_{3}\right): \delta_{\mathrm{H}} 7.43(\mathrm{~d}, 2 \mathrm{H}, J 5 \mathrm{~Hz}$, arom), $7.12(\mathrm{~d}, 2 \mathrm{H}, \mathrm{J} 5 \mathrm{~Hz}$, arom), 5.64 (s, 1H, H-4'), 4.77, 4.63 (both br s, 2H, H-29), 3.93 (s, 2H, CH $\mathrm{CH}_{2}$ ') $^{2} 3.70$ (s, 3H, OMe), $3.02(\mathrm{~m}, 1 \mathrm{H}, \mathrm{H}-19)$, $2.35\left(\mathrm{~d}, 1 \mathrm{H}, J 15.0 \mathrm{~Hz}, \mathrm{H}^{\mathrm{a}}-1\right), 2.27-1.25\left(\mathrm{~m}, 21 \mathrm{H}, \mathrm{CH}, \mathrm{CH}_{2}\right.$ in pentacyclic skeleton and $\left.1 \mathrm{H}, \mathrm{H}^{\mathrm{b}}-1\right), 1.72(\mathrm{~s}, 3 \mathrm{H}, \mathrm{H}-$ 30), 1.20, 1.10, 1.00, 0.98, 0.85 (all s, 3H each, H-23- H-27). $\left.{ }^{13} \mathrm{C} \mathrm{NMR} \mathrm{(100} \mathrm{MHz,} \mathrm{CDCl}\right): \delta_{c} 176.68,155.40$, $151.39,150.59,137.75,131.41,130.48,128.75,113.74,109.60,107.82,56.50,53.56,51.28,49.44,49.19$, 46.97, 42.41, 40.72, 38.59, 38.59, 38.39, 36.96, 34.66, 34.15, 33.14, 32.14, 30.62, 29.78, 29.10, 25.61, 21.36, 21.36, 19.39, 18.95, 16.32, 15.69, 14.69. Anal. Calcd for $\mathrm{C}_{40} \mathrm{H}_{53} \mathrm{BrO}_{3}$ : C, 72.60; H, 8.07. Found: C, 72.54; $\mathrm{H}$, 8.16\%. MS (APCI): $m / z[\mathrm{M}+\mathrm{H}]^{+}$, found 661. $\left[\mathrm{C}_{40} \mathrm{H}_{53} \mathrm{O}_{3} \mathrm{Br}\right]^{+}$requires 660.32 .

Methyl 5' -(4-chlorobenzyl)furano[3,2-b]lup-20(29)-en-28-oate (15c). Colorless crystals (70\%). mp 180-183 ${ }^{\circ} \mathrm{C}$. $[\alpha]_{\mathrm{D}}^{21}+23.47^{\circ}\left(c 0.70, \mathrm{CHCl}_{3}\right) .{ }^{1} \mathrm{H} \mathrm{NMR}\left(500 \mathrm{MHz}, \mathrm{CDCl}_{3}\right): \delta_{\mathrm{H}} 7.27(\mathrm{~d}, 2 \mathrm{H}, J 5 \mathrm{~Hz}$, arom), 7.18 (d, 2H, J $5 \mathrm{~Hz}$, arom), $5.64\left(\mathrm{~s}, 1 \mathrm{H}, \mathrm{H}-4^{\prime}\right), 4.77,4.64$ (both br s, 2H, H-29), 3.89 (s, 2H, CH $\mathrm{CH}_{2}$ ) , 3.70 (s, 3H, OMe), 3.04 (m, 1H, H-19), $2.36\left(\mathrm{~d}, 1 \mathrm{H}, J 15.0 \mathrm{~Hz}, \mathrm{H}^{\mathrm{a}}-1\right), 2.34-1.23\left(\mathrm{~m}, 21 \mathrm{H}, \mathrm{CH}, \mathrm{CH}_{2}\right.$ in pentacyclic skeleton and $\left.1 \mathrm{H}, \mathrm{H}^{\mathrm{b}}-1\right), 1.72(\mathrm{~s}, 3 \mathrm{H}, \mathrm{H}-$ 30), 1.21, 1.11, 1.00, 0.99, 0.85 (all s, 3H each, H-23-H-27). $\left.{ }^{13} \mathrm{C} \mathrm{NMR} \mathrm{(125} \mathrm{MHz,} \mathrm{CDCl}_{3}\right): \delta_{\mathrm{C}} 176.68,155.37$, $151.52,150.58,137.22,132.02,130.08,128.46,113.74,109.62,107.79,56.50,53.55,51.29,49.44,49.19$, $46.97,42.41,40.72,38.59,38.56,38.39,36.97,34.66,34.09,33.48,32.14,30.62,29.78,29.10,25.61,21.37$, 21.37, 19.40, 18.96, 16.33, 15.70, 14.70. Anal. Calcd for $\mathrm{C}_{40} \mathrm{H}_{53} \mathrm{ClO}_{3}$ : C, 77.83; $\mathrm{H}, 8.65$. Found: $\mathrm{C}, 77.89 ; \mathrm{H}, 8.6 \%$. $\mathrm{MS}(\mathrm{APCl}): \mathrm{m} / z[\mathrm{M}+\mathrm{H}]^{+}$, found 617. $\left[\mathrm{C}_{40} \mathrm{H}_{53} \mathrm{O}_{3} \mathrm{Cl}\right]^{+}$requires 616.37 .

Methyl 5'-(4-fluorobenzyl)furano[3,2-b]lup-20(29)-en-28-oate (15d). Colorless crystals (72\%). mp 77-79 ${ }^{\circ} \mathrm{C}$. $[\alpha]_{\mathrm{D}}^{21}+10.3^{\circ}$ (c 1.97, $\mathrm{CHCl}_{3}$ ). ${ }^{1} \mathrm{H}$ NMR $\left(500 \mathrm{MHz}^{\mathrm{C}} \mathrm{CDCl}_{3}\right): \delta_{\mathrm{H}}$ 7.22-7.19 (m, 2H, arom), 7.02-6.97 (m, 2H, arom), $5.63\left(\mathrm{~s}, 1 \mathrm{H}, \mathrm{H}-4^{\prime}\right), 4.78,4.64$ (both br s, 2H, H-29), 3.90 (s, 2H, CH2-5'), 3.70 (s, 3H, OMe), 3.05 (m, 1H, H-19), $2.35\left(\mathrm{~d}, 1 \mathrm{H}, J 15.0 \mathrm{~Hz}, \mathrm{H}^{\mathrm{a}}-1\right), 2.28-1.09\left(\mathrm{~m}, 21 \mathrm{H}, \mathrm{CH}, \mathrm{CH}_{2}\right.$ in pentacyclic skeleton and $\left.1 \mathrm{H}, \mathrm{H}^{\mathrm{b}}-1\right), 1.72(\mathrm{~s}, 3 \mathrm{H}, \mathrm{H}-$ 30), 1.23, 1.21, 1.01, 0.99, 0.86 (all s, 3H each, $\mathrm{H}-23-\mathrm{H}-27) .{ }^{13} \mathrm{C} \mathrm{NMR}\left(125 \mathrm{MHz}, \mathrm{CDCl}_{3}\right): \delta_{\mathrm{c}} 176.67,162.75$, $160.32,155.28,151.97,150.58,134.38,130.17,130.09,115.21,115.00,113.71,109.62,107.63,51.27,56.60$, 53.57, 49.45, 49.20, 46.97, 42.41, 40.72, 38.59, 38.59, 38.39, 36.97, 34.66, 33.93, 33.49, 32.14, 30.62, 29.79, 29.10, 25.62, 21.37, 21.37, 19.40, 18.96, 16.33, 15.70, 14.70. Anal. Calcd for $\mathrm{C}_{40} \mathrm{H}_{53} \mathrm{FO}_{3}: \mathrm{C}, 79.96 ; \mathrm{H}, 8.89 \%$. Found: $\mathrm{C}, 79.84 ; \mathrm{H}, 8.82 . \mathrm{MS}: \mathrm{m} / \mathrm{z}[\mathrm{M}-\mathrm{H}]^{+}$, found $599.31\left[\mathrm{C}_{40} \mathrm{H}_{53} \mathrm{O}_{3} \mathrm{~F}\right]^{+}$requires 600.40 .

Methyl 5'-(2-methylbenzyl)furano[3,2-b]lup-20(29)-en-28-oate (15e). Colorless crystals (77\%). mp 63-65 ${ }^{\circ} \mathrm{C}$. $[\alpha]_{\mathrm{D}}^{21}+21.76^{\circ}\left(\mathrm{c} 0.60, \mathrm{CHCl}_{3}\right) .{ }^{1} \mathrm{H}$ NMR $\left(500 \mathrm{MHz}, \mathrm{CDCl}_{3}\right): \delta_{\mathrm{H}} 7.18-7.15(\mathrm{~m}, 4 \mathrm{H}$, arom $), 5.57\left(\mathrm{~s}, 1 \mathrm{H}, \mathrm{H}-4{ }^{\prime}\right), 4.80,4.66$ (both br s, 2H, H-29), 3.92 (s, 2H, CH${ }_{2}^{-5}$ ), 3.72 (s, 3H, OMe), 3.07 (m, 1H, H-19), $2.37\left(\mathrm{~m}, 1 \mathrm{H}, \mathrm{H}^{\mathrm{a}}-1\right), 2.35$ (s, 3H, 
Me- $\left.\mathrm{C}_{6} \mathrm{H}_{4}\right), 2.30-1.25\left(\mathrm{~m}, 21 \mathrm{H}, \mathrm{CH}, \mathrm{CH}_{2}\right.$ in pentacyclic skeleton and $\left.1 \mathrm{H}, \mathrm{H}^{\mathrm{b}}-1\right), 1.74(\mathrm{~s}, 3 \mathrm{H}, \mathrm{H}-30), 1.25,1.14$, 1.03,1.01, 0.88 (all s, 3H each, H-23-H-27). ${ }^{13} \mathrm{C}$ NMR (125 MHz, CDCl $): \delta_{c} 176.67,154.91,151.76,150.56$, 137.10, 136.43, 130.12, 129.42, 126.50, 125.99, 113.71, 109.67, 107.53, 56.62, 53.59, 51.30, 49.47, 49.21, $46.99,42.43,40.74,38.63,38.63,38.41,36.99,34.67,33.53,32.50,32.17,30.65,29.82,29.18,25.65,21.45$, 21.41, 19.49, 19.44, 19.00, 16.38, 15.73, 14.73. Anal. Calcd for $\mathrm{C}_{41} \mathrm{H}_{56} \mathrm{O}_{3}$ : C, 82.53; $\mathrm{H}, 9.49$. Found: $\mathrm{C}, 82.49 ; \mathrm{H}$, $9.41 \%$. MS (APCI): $m / z[\mathrm{M}+\mathrm{H}]^{+}$, found 597. $\left[\mathrm{C}_{41} \mathrm{H}_{56} \mathrm{O}_{3}\right]^{+}$requires 596.42 .

Methyl 5'-(3,4,5-trimethoxybenzyl)furano[3,2-b]lup-20(29)-en-28-oate (15f). Colorless crystals (73\%). mp 83$86^{\circ} \mathrm{C} .[\alpha]_{\mathrm{D}}^{20}+18.6^{\circ}$ (c $\left.0.85, \mathrm{CHCl}_{3}\right) .{ }^{1} \mathrm{H}$ NMR $\left(500 \mathrm{MHz}, \mathrm{CDCl}_{3}\right): \delta_{\mathrm{H}} 7.47\left(\mathrm{~s}, 2 \mathrm{H}\right.$, arom) $, 5.71\left(\mathrm{~s}, 1 \mathrm{H}, \mathrm{H}-4{ }^{\prime}\right), 4.77,4.63$ (both br s, 2H, H-29), 3.88 (s, 2H, CH${ }_{2}-5$ ) , 3.84 (s, 9H, Ph(OMe) 3 ), 3.69 (s, 3H, OMe), 3.04 (m, 1H, H-19), 2.35 $\left(\mathrm{d}, 1 \mathrm{H}, \mathrm{J} 15 \mathrm{~Hz}, \mathrm{H}^{\mathrm{a}}-1\right), 2.28-1.24\left(\mathrm{~m}, 21 \mathrm{H}, \mathrm{CH}, \mathrm{CH}_{2}\right.$ in pentacyclic skeleton and $\left.1 \mathrm{H} \mathrm{H}^{\mathrm{b}}-1\right), 1.71(\mathrm{~s}, 3 \mathrm{H}, \mathrm{H}-30), 1.23$, 1.12, 1.00, 0.98, 0.86 (all s, 3H each, $\mathrm{H}-23-\mathrm{H}-27) .{ }^{13} \mathrm{C} N M R\left(125 \mathrm{MHz} \mathrm{CDCl}_{3}\right): \delta_{\mathrm{C}} 176.66,155.26,153.09$, $151.79,150.56,136.32,134.57,113.76,109.62,107.78,105.50,60.84,56.59,55.98,53.55,51.28,49.43$, 49.20, 46.96, 42.41, 40.72, 38.61, 38.59, 38.38, 36.96, 34.82, 33.67, 33.48, 32.13, 30.61, 29.78, 29.15, 25.61, 21.40, 21.40, 19.40, 18.97, 16.33, 15.70, 14.70. Anal. Calcd for $\mathrm{C}_{43} \mathrm{H}_{60} \mathrm{O}_{6}: \mathrm{C}, 76.56 ; \mathrm{H}, 8.87$. Found: $\mathrm{C}, 76.49 ; \mathrm{H}$, $8.85 \%$. MS: $m / z[\mathrm{M}-\mathrm{H}]^{+}$, found $671.34\left[\mathrm{C}_{43} \mathrm{H}_{60} \mathrm{O}_{6}\right]^{+}$requires 672.44 .

Methyl 5'-(4-nitrobenzyl)furano[3,2-b]lup-20(29)-en-28-oate (15g). Colorless crystals (19\%). mp 60-62 ${ }^{\circ} \mathrm{C}$. $[\alpha]_{\mathrm{D}}^{21}+19.0^{\circ}$ (c 0.54, $\left.\mathrm{CHCl}_{3}\right) .{ }^{1} \mathrm{H}$ NMR $\left(400 \mathrm{MHz}, \mathrm{CDCl}_{3}\right): \delta_{\mathrm{H}} 8.15(\mathrm{~d}, 2 \mathrm{H}, J 5 \mathrm{~Hz}$, arom), $7.39(\mathrm{~d}, 2 \mathrm{H}, J 5 \mathrm{~Hz}, \operatorname{arom})$, $5.73\left(\mathrm{~s}, 1 \mathrm{H}, \mathrm{H}-4^{\prime}\right), 4.77,4.63$ (both br s, 2H, H-29), 4.03 (s, 2H, CH $\mathrm{C}_{2}-5$ ), 3.69 (s, 3H, OMe), $3.04(\mathrm{~m}, 1 \mathrm{H}, \mathrm{H}-19)$, $2.36\left(\mathrm{~d}, 1 \mathrm{H}, J 15 \mathrm{~Hz}, \mathrm{H}^{\mathrm{a}}-1\right), 1.71(\mathrm{~s}, 3 \mathrm{H}, \mathrm{H}-30), 2.27-1.25\left(\mathrm{~m}, 21 \mathrm{H}, \mathrm{CH}, \mathrm{CH}_{2}\right.$ in pentacyclic skeleton and $\left.1 \mathrm{H}, \mathrm{H}^{\mathrm{b}}-1\right)$, 1.19, 1.10, 1.00, 0.98, 0.85 (all s, 3H each, H-23-H-27). ${ }^{13} \mathrm{C} \mathrm{NMR} \mathrm{(100} \mathrm{MHz,} \mathrm{CDCl} 3$ ): $\delta_{\mathrm{C}} 176.68,155.94,150.59$, 149.87, 146.54, 146.54, 129.44, 123.67, 113.92, 109.61, 108.49, 56.59, 53.51, 51.29, 49.42, 49.18, 46.95, $42.41,40.71,38.59,38.50,38.37,36.96,34.68,34.56,33.44,32.12,30.60,29.77,29.07,25.59,21.35,21.35$, 19.39, 18.93, 16.32, 15.69, 14.68. Anal. Calcd for $\mathrm{C}_{40} \mathrm{H}_{53} \mathrm{NO}_{5}$ : C, 76.52; $\mathrm{H}, 8.51$. Found: $\mathrm{C}, 76.57 ; \mathrm{H}, 8.50 \%$. MS (APCl): $m / z[\mathrm{M}+\mathrm{H}]^{+}$, found $628 .\left[\mathrm{C}_{40} \mathrm{H}_{53} \mathrm{NO}_{5}\right]^{+}$requires 627.39.

Methyl 5'-benzylfurano[3,2-b]urs-12-en-28-oate (18a). Colorless crystals (71\%), mp 76-78 ${ }^{\circ} \mathrm{C} .[\alpha]_{\mathrm{D}}^{19}+64.8^{\circ}(c$ 0.62, $\left.\mathrm{CHCl}_{3}\right) .{ }^{1} \mathrm{H}$ NMR $\left(400 \mathrm{MHz}, \mathrm{CDCl}_{3}\right): \delta_{\mathrm{H}} 7.35-7.25(\mathrm{~m}, 5 \mathrm{H}, \mathrm{arom}), 5.67\left(\mathrm{~s}, 1 \mathrm{H}, \mathrm{H}-4{ }^{\prime}\right), 5.35(\mathrm{~m}, 1 \mathrm{H}, \mathrm{H}-12), 3.96$ (s, 2H, CH $\left.{ }_{2}-5^{\prime}\right), 3.65$ (s, 3H, OMe), 2.35 (d, 1H, J $\left.15 \mathrm{~Hz}, \mathrm{H}^{\mathrm{a}}-1\right), 2.30(\mathrm{~m}, 1 \mathrm{H}, \mathrm{H}-18), 2.12-1.07\left(\mathrm{~m}, 19 \mathrm{H}, \mathrm{CH}, \mathrm{CH}_{2}\right.$ in pentacyclic skeleton and $\left.1 \mathrm{H}, \mathrm{H}^{\mathrm{b}}-1\right), 1.26,1.16,1.14,0.98,0.85$ (all s, 3H each, $\left.\mathrm{H}-23-\mathrm{H}-27\right), 0.99(\mathrm{~d}, 3 \mathrm{H}, \mathrm{J} 5 \mathrm{~Hz}$, $\mathrm{H}-30), 0.93$ (d, 3H, J 5Hz, H-30). ${ }^{13} \mathrm{C} N M R\left(100 \mathrm{MHz}, \mathrm{CDCl}_{3}\right): \delta_{\mathrm{C}} 178.06,155.08,152.25,138.75,138.08,128.75$, 128.37, 126.23, 124.58, 113.64, 107.51, 53.56, 53.07, 51.46, 48.21, 46.13, 42.15, 39.61, 39.17, 38.92, 38.35, $38.35,36.68,34.75,34.57,32.44,30.73,29.34,28.12,24.32,23.52,23.37,21.53,21.21,19.04,17.09,16.82$, 15.74. Anal. Calcd for $\mathrm{C}_{40} \mathrm{H}_{54} \mathrm{O}_{3}$ : C, 82.43; H, 9.34. Found: $\mathrm{C}, 82.39 ; \mathrm{H}, 9.37 \%$. MS (APCl): $m / z[M+H]^{+}$, found 583. $\left[\mathrm{C}_{40} \mathrm{H}_{54} \mathrm{O}_{3}\right]^{+}$requires 582.41 .

Methyl 5'-benzylfurano[3,2-b]olean-12-en-28-oate (20a). Colorless crystals (72\%). mp 76-78 ${ }^{\circ} \mathrm{C} .[\alpha]_{\mathrm{D}}^{20}+81.0^{\circ}(c$ 0.70, $\left.\mathrm{CHCl}_{3}\right) .{ }^{1} \mathrm{H} N M R\left(400 \mathrm{MHz}, \mathrm{CDCl}_{3}\right): \delta_{\mathrm{H}} 7.34-7.31\left(\mathrm{~m}, 5 \mathrm{H}\right.$, arom) $5.66\left(\mathrm{~s}, 1 \mathrm{H}, \mathrm{H}-4{ }^{\prime}\right), 5.38(\mathrm{~m}, 1 \mathrm{H}, \mathrm{H}-12), 3.95$ (s, 2H, CH $\left.\mathrm{CH}_{2} 5^{\prime}\right), 3.67$ (s, 3H, OMe), $2.93(\mathrm{~m}, 1 \mathrm{H}, \mathrm{H}-18), 2.32\left(\mathrm{~d}, 1 \mathrm{H}, \mathrm{J} 15 \mathrm{~Hz}, \mathrm{H}^{\mathrm{a}}-1\right), 2.02-1.22\left(\mathrm{~m}, 19 \mathrm{H}, \mathrm{CH}, \mathrm{CH}_{2}\right.$ in pentacyclic skeleton and $1 \mathrm{H}, \mathrm{H}^{\mathrm{b}}-1$ ), 1.25, 1.19, 1.16, 0.96, 0.83 (all s, 3H each, H-23-H-27), 0.98 (s, 3H, H-30), 0.94 (s, 3H, H-30). ${ }^{13} \mathrm{C} N M R\left(100 \mathrm{MHz}, \mathrm{CDCl}_{3}\right): \delta_{\mathrm{C}} 178.30,155.12,152.27,143.66,138.74,128.75,128.36$, $126.23,122.53,113.59,107.51,53.53,51.55,46.82,46.20,45.92,41.82,41.46,39.41,38.40,38.11,34.74$, 34.56, 33.92, 33.15, 32.41, 32.12, 30.73, 29.31, 27.79, 25.77, 23.71, 23.66, 23.40, 21.54, 19.06, 16.64, 15.56. Anal. Calcd for $\mathrm{C}_{40} \mathrm{H}_{54} \mathrm{O}_{3}$ : C, 82.43; $\mathrm{H}, 9.34$. Found: $\mathrm{C}, 82.39 ; \mathrm{H}, 9.28 \%$. MS (APCI): $\mathrm{m} / z[\mathrm{M}+\mathrm{H}]^{+}$, found 583 . $\left[\mathrm{C}_{40} \mathrm{H}_{54} \mathrm{O}_{3}\right]^{+}$requires 582.41 .

Halogenolysis of compounds 11a-13a, 15a-f, 18a and 20a with Lil in DMF. Lil (0.85 g, $6.5 \mathrm{mmol})$ was added to a stirred solution of compound 11a-13a, 15a-f, 18a or $20 a(0.4 \mathrm{mmol})$ in DMF (3 mL). The reaction mixture was 
heated under reflux for $5 \mathrm{~h}$ (monitoring by TLC), diluted with water $(2 \mathrm{~mL})$, and neutralized with $5 \% \mathrm{HCl}(\mathrm{aq})$. The product was extracted with EtOAc $(3 \times 10 \mathrm{~mL})$. The combined extracts were dried with $\mathrm{MgSO}_{4}$ and concentrated. The residue was purified by column chromatography on $\mathrm{SiO}_{2}$ with hexane/EtOAc (from 30:1 to $1: 10)$ as an eluent to give compound $\mathbf{1 1 b}-\mathbf{1 3 b}, \mathbf{1 6 a - f ,} \mathbf{1 8 b}$ and $\mathbf{2 0 b}$.

5'-Methylfurano[3,2-b]lup-20(29)-en-28-oic acid (11b). Colorless crystals (45\%). mp 142-144 ${ }^{\circ} \mathrm{C} .[\alpha]_{\mathrm{D}}^{19}+31.4^{\circ}(c$ 0.77, $\mathrm{CHCl}_{3}$ ). ${ }^{1} \mathrm{H}$ NMR $\left(500 \mathrm{MHz}, \mathrm{CDCl}_{3}\right): \delta_{\mathrm{H}} 5.69(\mathrm{~s}, 1 \mathrm{H}, \mathrm{H}-4$ '), 4.79, 4.66 (both br s, 2H, H-29), $3.07(\mathrm{~m}, 1 \mathrm{H}, \mathrm{H}-$ 19), $2.37\left(\mathrm{~d}, 1 \mathrm{H}, J 15 \mathrm{~Hz}, \mathrm{H}^{\mathrm{a}}-1\right), 2.26\left(\mathrm{~s}, 3 \mathrm{H}, \mathrm{Me}-5^{\prime}\right), 1.70(\mathrm{~s}, 3 \mathrm{H}, \mathrm{H}-30), 2.32-1.27\left(\mathrm{~m}, 21 \mathrm{H}, \mathrm{CH}, \mathrm{CH}_{2}\right.$ in pentacyclic skeleton and $\left.1 \mathrm{H}, \mathrm{H}^{\mathrm{b}}-1\right), 1.22,1.11,1.04,1.02,0.88$ (all s, 3H each, $\left.\mathrm{H}-23-\mathrm{H}-27\right) .{ }^{13} \mathrm{C} \mathrm{NMR}\left(125 \mathrm{MHz}_{2} \mathrm{CDCl}_{3}\right): \delta_{\mathrm{C}}$ 183.26, 154.44, 150.38, 149.60, 113.66, 109.76, 106.78, 56.53, 53.60, 49.29, 49.16, 46.96, 42.45, 40.73, 38.64, $38.62,38.59,37.07,34.61,33.49,32.16,30.62,29.84,29.17,25.60,21.36,21.36,19.42,18.94,16.31,15.82$, 14.72, 13.73. Anal. Calcd for $\mathrm{C}_{33} \mathrm{H}_{48} \mathrm{O}_{3}: \mathrm{C}, 80.44 ; \mathrm{H}, 9.82$. Found: C, 80.49; $\mathrm{H}, 9.86 \%$. MS (APCl): $m / z$ [M-H]', found 491. $\left[\mathrm{C}_{33} \mathrm{H}_{48} \mathrm{O}_{3}\right]^{+}$requires 492.36 .

5'-Methylfurano[3,2-b]urs-12-en-28-oic acid (12b). Colorless crystals (65\%). mp 160-163 ${ }^{\circ} \mathrm{C}$. $[\alpha]_{\mathrm{D}}^{19}+80.4^{\circ}$ (c 0.92, $\left.\mathrm{CHCl}_{3}\right) .1 \mathrm{H}$ NMR $\left(500 \mathrm{MHz}, \mathrm{CDCl}_{3}\right): \delta_{\mathrm{H}} 5.69\left(\mathrm{~s}, 1 \mathrm{H}, \mathrm{H}-4{ }^{\prime}\right), 5.32(\mathrm{~m}, 1 \mathrm{H}, \mathrm{H}-12), 2.35\left(\mathrm{~d}, 1 \mathrm{H}, J 15 \mathrm{~Hz}, \mathrm{H}^{\mathrm{a}}-1\right)$, $2.29(\mathrm{~m}, 1 \mathrm{H}, \mathrm{H}-18), 2.26\left(\mathrm{~s}, 3 \mathrm{H}, \mathrm{Me}-5^{\prime}\right), 2.07-1.05\left(\mathrm{~m}, 19 \mathrm{H}, \mathrm{CH}, \mathrm{CH} 2\right.$ in pentacyclic skeleton and $\left.1 \mathrm{H}, \mathrm{H}^{\mathrm{b}}-1\right), 1.22$, 1.12, 1.10, 0.96, 0.85 (all s, 3H each, H-23-H-27), 0.97 (d, 3H, J 5Hz, H-30), 0.90 (d, 3H, J 5Hz, H-30). ${ }^{13} \mathrm{C} \mathrm{NMR}$ $\left(125 \mathrm{MHz}, \mathrm{CDCl}_{3}\right): \delta_{\mathrm{C}} 184.42,154.42,149.65,137.77,125.99,113.58,106.65,53.55,52.63,48.10,46.12,42.06$, $39.57,39.11,38.84,38.37,38.30,36.75,34.47,32.39,30.66,29.31,28.05,24.10,23.50,23.34,21.52,21.18$, $18.93,16.99,16.96,15.66,13.73$. Anal. Calcd for $\mathrm{C}_{33} \mathrm{H}_{48} \mathrm{O}_{3}: \mathrm{C}, 80.44 ; \mathrm{H}, 9.82$. Found: $\mathrm{C}, 80.51 ; \mathrm{H}, 9.85 \%$. MS: $\mathrm{m} / \mathrm{z}[\mathrm{M}+\mathrm{H}]^{+}$, found $493.07\left[\mathrm{C}_{33} \mathrm{H}_{48} \mathrm{O}_{3}\right]^{+}$requires 492.36 .

5'-Methylfurano[3,2-b]olean-12-en-28-oic acid (13b). Colorless crystals (48\%). mp 138-140 ${ }^{\circ} \mathrm{C} .[\alpha]_{\mathrm{D}}^{19}+84.2^{\circ}(\mathrm{c}$ 0.84, $\mathrm{CHCl}_{3}$ ). $1 \mathrm{H}$ NMR (500 MHz, CDCl 3$): \delta_{\mathrm{H}} 5.69(\mathrm{~s}, 1 \mathrm{H}, \mathrm{H}-4$ '), $5.36(\mathrm{~m}, 1 \mathrm{H}, \mathrm{H}-12), 2.88(\mathrm{~m}, 1 \mathrm{H}, \mathrm{H}-18), 2.31(\mathrm{~d}$, $\left.1 \mathrm{H}, \mathrm{J} 15 \mathrm{~Hz}, \mathrm{H}^{\mathrm{a}}-1\right), 2.26\left(\mathrm{~s}, 3 \mathrm{H}, \mathrm{Me}-5^{\prime}\right), 2.07-1.29\left(\mathrm{~m}, 19 \mathrm{H}, \mathrm{CH}, \mathrm{CH}_{2}\right.$ in pentacyclic skeleton and $\left.1 \mathrm{H}, \mathrm{H}^{\mathrm{b}}-1\right), 1.22$, $1.18,1.11,0.95,0.84$ (all s, 3H each, H-23-H-27), 0.96 (s, 3H, H-30), 0.93 (s, 3H, H-30). ${ }^{13} \mathrm{C} \mathrm{NMR} \mathrm{(125} \mathrm{MHz,}$ $\left.\mathrm{CDCl}_{3}\right): \delta_{c} 184.58,154.45,149.66,143.42,122.77,113.54,106.65,53.51,46.66,46.22,45.87,41.74,41.03$, $39.38,38.39,38.09,34.46,33.84,33.08,32.45,32.05,30.69,29.30,27.74,25.78,23.57,23.38,22.95,21.53$, 18.96, 16.86, 15.49, 13.73. Anal. Calcd for $\mathrm{C}_{33} \mathrm{H}_{48} \mathrm{O}_{3}: \mathrm{C}, 80.44 ; \mathrm{H}, 9.82$. Found: $\mathrm{C}, 80.50 ; \mathrm{H}, 9.83 \%$. MS: $\mathrm{m} / \mathrm{z}$ $[\mathrm{M}+\mathrm{H}]^{+}$, found $493.03\left[\mathrm{C}_{33} \mathrm{H}_{48} \mathrm{O}_{3}\right]^{+}$requires 492.36 .

5'-Benzylfurano[3,2-b]lup-20(29)-en-28-oic acid (16a). Colorless crystals (45\%). mp 252-254 ${ }^{\circ} \mathrm{C} .[\alpha]_{\mathrm{D}}^{19}+28.6^{\circ}(c$ 0.63, $\mathrm{CHCl}_{3}$ ). ${ }^{1} \mathrm{H}$ NMR $\left(500 \mathrm{MHz}, \mathrm{CDCl}_{3}\right): \delta_{\mathrm{H}} 7.33-7.22(\mathrm{~m}, 5 \mathrm{H}$, arom), $5.63(\mathrm{~s}, 1 \mathrm{H}, \mathrm{H}-4$ '), 4.78, 4.65 (both br s, 2H, $\mathrm{H}-29), 3.94$ (s, 2H, CH$\left.{ }_{2}-5^{\prime}\right), 3.05$ (m, 1H, H-19), 2.35 (d, 1H, J $\left.15.0 \mathrm{~Hz}, \mathrm{H}^{\mathrm{a}}-1\right), 1.73$ (s, 3H, H-30), 2.33-1.27 (m, $21 \mathrm{H}, \mathrm{CH}, \mathrm{CH}_{2}$ in pentacyclic skeleton and $1 \mathrm{H}, \mathrm{H}^{\mathrm{b}}-1$ ), 1.22, 1.11, 1.02, 1.01, 0.86 (all s, 3H each, $\mathrm{H}-23-\mathrm{H}-27$ ). ${ }^{13} \mathrm{C}$ NMR $\left(125 \mathrm{MHz}_{\mathrm{CDCl}}\right)$ : $\delta_{\mathrm{c}} 182.47,155.10,152.23,150.42,138.73,128.76,128.36,126.22,113.67,109.73$, 107.57, 56.48, 53.55, 49.26, 49.12, 46.93, 42.44, 40.72, 38.57, 38.57, 38.57, 37.05, 34.74, 34.66, 33.46, 32.14, $30.59,29.82,29.12,25.58,21.34,21.34,19.40,18.92,16.33,15.81,14.69$. Anal. Calcd for $\mathrm{C}_{39} \mathrm{H}_{52} \mathrm{O}_{3}: \mathrm{C}, 82.35 ; \mathrm{H}$, 9.21. Found: $\mathrm{C}, 82.31 ; \mathrm{H}, 9.18 \%$. MS (APCI): $\mathrm{m} / \mathrm{z}[\mathrm{M}-\mathrm{H}]^{-}$, found 567. $\left[\mathrm{C}_{39} \mathrm{H}_{52} \mathrm{O}_{3}\right]^{+}$requires 568.39 .

5'-(4-Brombenzyl)furano[3,2-b]lup-20(29)-en-28-oic acid (16b). Colorless crystals (43\%). mp 230-232 ${ }^{\circ} \mathrm{C} .[\alpha]_{\mathrm{D}}^{19}$

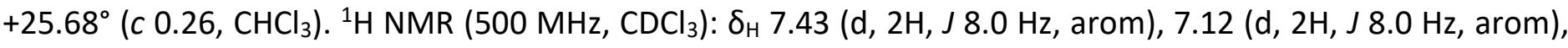
5.64 (s, $\left.1 \mathrm{H}, \mathrm{H}-4^{\prime}\right), 4.77,4.65$ (both br s, 2H, H-29), 3.87 (s, 2H, $\mathrm{CH}_{2}-5$ '), $3.05(\mathrm{~m}, 1 \mathrm{H}, \mathrm{H}-19), 2.35$ (d, $1 \mathrm{H}, J 12.0 \mathrm{~Hz}$, $\left.\mathrm{H}^{\mathrm{a}}-1\right), 2.33-1.25\left(\mathrm{~m}, 2 \mathrm{H}, \mathrm{CH}, \mathrm{CH}_{2}\right.$ in pentacyclic skeleton), $1.92\left(\mathrm{~d}, 1 \mathrm{H}, J 12.0 \mathrm{~Hz}, \mathrm{H}^{\mathrm{b}}-1\right), 1.77(\mathrm{~s}, 3 \mathrm{H}, \mathrm{H}-30), 1.20$ 1.09, 1.01, 1.00, 0.85 (all s, 3H each, H-23-H-27). ${ }^{13} \mathrm{C} \mathrm{NMR}\left(125 \mathrm{MHz}, \mathrm{CDCl}_{3}\right): \delta_{\mathrm{c}} 182.29,155.38,151.43,150.40$, $137.74,131.43,130.49,120.08,113.73,109.74,107.80,56.46,53.52,49.25,49.11,46.93,42.44,40.72,38.59$, $38.56,38.56,37.04,34.66,34.15,33.44,32.13,30.58,29.81,29.10,25.57,21.33,21.33,19.40,18.92,16.33$, 15.80, 14.68. Anal. Calcd for $\mathrm{C}_{39} \mathrm{H}_{51} \mathrm{BrO}_{3}$ : C, 72.32; $\mathrm{H}, 7.94$. Found: $\mathrm{C}, 72.28 ; \mathrm{H}, 7.96 \%$. 
5'-(4-Chlorobenzyl)furano[3,2-b]lup-20(29)-en-28-oic acid (16c). Colorless crystals (45\%). mp 263-265 ${ }^{\circ} \mathrm{C}$. $[\alpha]_{\mathrm{D}}^{19}$ $+25.71^{\circ}$ (c 0.32, $\mathrm{CHCl}_{3}$ ). ${ }^{1} \mathrm{H} \mathrm{NMR}\left(500 \mathrm{MHz} \mathrm{CDCl}_{3}\right): \delta_{\mathrm{H}} 7.28(\mathrm{~d}, 2 \mathrm{H}, J 8.0 \mathrm{~Hz}$, arom), $7.18(\mathrm{~d}, 2 \mathrm{H}, J 8.0 \mathrm{~Hz}$, arom),

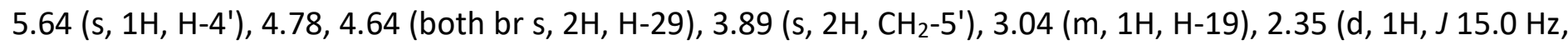
$\left.\mathrm{H}^{\mathrm{a}}-1\right), 2.30-1.25\left(\mathrm{~m}, 2 \mathrm{H}, \mathrm{CH}, \mathrm{CH}_{2}\right.$ in pentacyclic skeleton), $1.91\left(\mathrm{~d}, 1 \mathrm{H}, J 15.0 \mathrm{~Hz}, \mathrm{H}^{\mathrm{b}}-1\right), 1.77$ (s, 3H, H-30), 1.20 1.09, 1.01, 1.00, 0.85 (all s, 3H each, $\mathrm{H}-23-\mathrm{H}-27) .{ }^{13} \mathrm{C} \mathrm{NMR}\left(125 \mathrm{MHz}, \mathrm{CDCl}_{3}\right.$ ): $\delta_{c}$ 182.30, 155.36, 151.55, 150.40, $137.21,132.02,130.08,128.46,113.72,109.74,107.77,56.46,53.52,49.25,49.12,46.92,42.44,40.72,38.60$, $38.60,38.56,37.04,34.66,34.09,33.44,32.13,30.58,29.81,29.10,25.57,21.33,21.33,19.40,18.92,16.33$, 15.80, 14.68. Anal. Calcd for $\mathrm{C}_{39} \mathrm{H}_{51} \mathrm{ClO}_{3}$ : C, 77.65; $\mathrm{H}, 8.52$. Found: $\mathrm{C}, 77.69 ; \mathrm{H}, 8.50 \%$.

5'-(4-Fluorobenzyl)furano[3,2-b]lup-20(29)-en-28-oic acid (16d). Colorless crystals (61\%). mp $268-2715{ }^{\circ} \mathrm{C}$. $[\alpha]_{\mathrm{D}}^{19}+26.4^{\circ}$ (c 0.80, $\mathrm{CHCl}_{3}$ ). ${ }^{1} \mathrm{H}$ NMR $\left(500 \mathrm{MHz}^{\mathrm{C}} \mathrm{CDCl}_{3}\right): \delta_{\mathrm{H}} 7.22-7.19(\mathrm{~m}, 2 \mathrm{H}$, arom), 7.01-6.98 (m, 2H, arom),

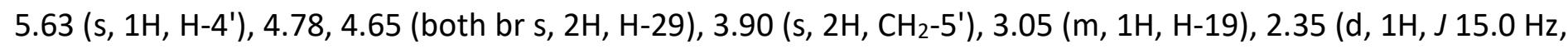
$\left.\mathrm{H}^{\mathrm{a}}-1\right), 2.28-1.25\left(\mathrm{~m}, 2 \mathrm{H}, \mathrm{CH}, \mathrm{CH}_{2}\right.$ in pentacyclic skeleton), $1.92\left(\mathrm{~d}, 1 \mathrm{H}, J 15.0 \mathrm{~Hz}, \mathrm{H}^{\mathrm{b}}-1\right), 1.73(\mathrm{~s}, 3 \mathrm{H}, \mathrm{H}-30), 1.21$ 1.10, 1.02, 1.01, 0.86 (all s, 3H each, $\mathrm{H}-23-\mathrm{H}-27) .{ }^{13} \mathrm{C} N M R\left(125 \mathrm{MHz}, \mathrm{CDCl}_{3}\right.$ ): $\delta_{\mathrm{C}} 182.54,162.50,160.57$, $155.26,152.01,150.40,134.34,130.17,130.10,115.19,115.02,113.69,109.74,107.61,56.48,53.53,49.25$, 49.11, 46.93, 42.43, 40.72, 38.60, 38.60, 38.57, 37.06, 34.66, 33.93, 33.45, 32.13, 30.59, 29.82, 29.10, 25.57, $21.33,21.33,19.40,18.91,16.33,15.80,14.68$. Anal. Calcd for $\mathrm{C}_{39} \mathrm{H}_{51} \mathrm{FO}_{3}: \mathrm{C}, 79.82 ; \mathrm{H}, 8.76$. Found: $\mathrm{C}, 79.92 ; \mathrm{H}$, $8.83 \%$.

5'-(4-Methylbenzyl)furano[3,2-b]lup-20(29)-en-28-oic acid (16e). Colorless crystals (45\%). mp 260-262 ${ }^{\circ} \mathrm{C}$. $[\alpha]_{\mathrm{D}}^{19}+21.6^{\circ}$ (c 0.245, $\left.\mathrm{CHCl}_{3}\right) .{ }^{1} \mathrm{H}$ NMR $\left(500 \mathrm{MHz}_{\mathrm{CDCl}}\right): \delta_{\mathrm{H}} 7.16-7.13\left(\mathrm{~m}, 4 \mathrm{H}\right.$, arom), $5.55\left(\mathrm{~s}, 1 \mathrm{H}, \mathrm{H}-4^{\prime}\right), 4.77$, 4.65 (both br s, 2H, H-29), $3.91\left(\mathrm{~s}, 2 \mathrm{H}, \mathrm{CH}_{2}-5^{\prime}\right), 3.05(\mathrm{~m}, 1 \mathrm{H}, \mathrm{H}-19), 2.35\left(\mathrm{~m}, 1 \mathrm{H}, \mathrm{H}^{\mathrm{a}}-1\right), 2.33\left(\mathrm{~s}, 3 \mathrm{H}, \mathrm{Me}^{-} \mathrm{C}_{6} \mathrm{H}_{4}\right)$, 2.33-1.25 (m, 20H, CH, CH in pentacyclic skeleton), 1.90 (d, $\left.1 \mathrm{H}, J 15.0 \mathrm{~Hz}, \mathrm{H}^{\mathrm{b}}-1\right), 1.76(\mathrm{~s}, 3 \mathrm{H}, \mathrm{H}-30), 1.22$ 1.05, 1.01, 1.00, 0.86 (all s, 3H each, H-23-H-27). ${ }^{13} \mathrm{C} N M R\left(125 \mathrm{MHz}, \mathrm{CDCl}_{3}\right): \delta_{\mathrm{C}} 182.18,154.90,151.76,150.41$, $137.09,136.45,130.10,129.41,126.47,125.96,113.68,109.73,107.48,56.46,53.53,49.25,49.11,46.92$, $42.44,40.72,38.60,38.61,38.56,37.04,34.65,33.47,32.46,32.13,30.59,29.81,29.14,25.58,21.37,21.36$, 19.45, 19.41, 18.93, 16.33, 15.81, 14.67. ${ }^{13} \mathrm{C}$ NMR (see Table 2). Anal. Calcd for $\mathrm{C}_{40} \mathrm{H}_{54} \mathrm{O}_{3}: \mathrm{C}, 82.43 ; \mathrm{H}, 9.34$. Found: $\mathrm{C}, 82.51 ; \mathrm{H}, 9.38 \%$.

5'-(3,4,5-Trimethoxybenzyl)furano[3,2-b]lup-20(29)-en-28-oic acid (16f). Colorless crystals (28\%). mp $210-212$ ${ }^{\circ} \mathrm{C} .[\alpha]_{\mathrm{D}}^{22}+22^{\circ}$ (c $\left.0.68, \mathrm{CHCl}_{3}\right) .{ }^{1} \mathrm{H}$ NMR $\left(500 \mathrm{MHz}, \mathrm{CDCl}_{3}\right): \delta_{\mathrm{H}} 6.48(\mathrm{~s}, 2 \mathrm{H}$, arom), $5.72(\mathrm{~s}, 1 \mathrm{H}, \mathrm{H}-4$ '), $4.78,4.65$ (both br s, 2H, H-29), 3.88 (s, 2H, CH${ }_{2}^{-5}$ ), 3.84 (s, 9H, Ph(OMe) 3 ), 3.06 (m, 1H, H-19), 2.36 (d, 1H, J 15 Hz, Ha -1), 2.34-1.28 (m, 20H, CH, CH2 in pentacyclic skeleton), $2.00\left(\mathrm{~d}, 1 \mathrm{H}, J 15 \mathrm{~Hz}, \mathrm{H}^{\mathrm{b}}-1\right), 1.73(\mathrm{~s}, 3 \mathrm{H}, \mathrm{H}-30), 1.24,1.12$, 1.03, 1.02, 0.87 (all s, 3H each, H-23-H-27). ${ }^{13} \mathrm{C} N M R\left(125 \mathrm{MHz}, \mathrm{CDCl}_{3}\right): \delta_{c}$ 183.07, 155.23, 153.09, 151.83, $150.38,136.32,134.58,113.75,109.76,107.77,105.52,60.85,56.51,55.98,53.53,49.26,49.13,46.95,42.44$, 40.72, 38.63, 38.59, 38.59, 37.06, 34.83, 33.68, 33.45, 32.14, 30.61, 29.83, 29.15, 25.57, 21.36, 21.36, 19.41, 18.93, 16.33, 15.82, 14.70. Anal. Calcd for $\mathrm{C}_{42} \mathrm{H}_{58} \mathrm{O}_{6}: \mathrm{C}, 76.56 ; \mathrm{H}, 8.87$. Found: $\mathrm{C}, 76.47 ; \mathrm{H}, 8.70 \%$. MS: $\mathrm{m} / \mathrm{z}$ [M$\mathrm{H}]^{+}$, found $657.41\left[\mathrm{C}_{42} \mathrm{H}_{58} \mathrm{O}_{6}\right]^{+}$requires 658.42 .

5'-Benzylfurano[3,2-b]urs-12-en-28-oic acid (18b). Colorless crystals (55\%), mp 126-129 ${ }^{\circ} \mathrm{C} .[\alpha]_{\mathrm{D}}^{19}+64.5^{\circ}(\mathrm{c}$ 0.95, $\left.\mathrm{CHCl}_{3}\right) .{ }^{1} \mathrm{H}$ NMR $\left(500 \mathrm{MHz}, \mathrm{CDCl}_{3}\right): \delta_{\mathrm{H}} 7.35-7.24(\mathrm{~m}, 5 \mathrm{H}, \mathrm{arom}), 5.67\left(\mathrm{~s}, 1 \mathrm{H}, \mathrm{H}-4{ }^{\prime}\right), 5.32(\mathrm{~m}, 1 \mathrm{H}, \mathrm{H}-12), 3.96$ (s, 2H, CH $\mathrm{CH}^{-}$) , 2.35 (d, 1H, J $\left.15 \mathrm{~Hz}, \mathrm{H}^{\mathrm{a}}-1\right), 2.25$ (d, 1H, J $\left.10 \mathrm{~Hz}, \mathrm{H}-18\right), 2.04\left(\mathrm{~m}, 1 \mathrm{H}, \mathrm{H}^{\mathrm{b}}-1\right), 2.01-1.07(\mathrm{~m}, 19 \mathrm{H}, \mathrm{CH}$, $\mathrm{CH}_{2}$ in pentacyclic skeleton), 1.24, 1.13, 1.12, 0.99, 0.87 (all s, 3H each, $\left.\mathrm{H}-23-\mathrm{H}-27\right), 0.98(\mathrm{~d}, 3 \mathrm{H}, J 5 \mathrm{~Hz}, \mathrm{H}-30)$, $0.91(\mathrm{~d}, 3 \mathrm{H}, J 5 \mathrm{~Hz}, \mathrm{H}-30) .{ }^{13} \mathrm{C} \mathrm{NMR}\left(125 \mathrm{MHz}, \mathrm{CDCl}_{3}\right): \delta_{\mathrm{c}} 184.54,155.09,152.27,138.76,137.80,128.76$, $128.37,126.25,125.99,113.61,107.50,53.52,52.62,48.11,46.12,42.06,39.59,39.12,38.85,38.35,38.35$, 36.76, 34.75, 34.54, 32.38, 30.67, 29.29, 28.07, 24.11, 23.51, 23.33, 21.52, 21.20, 18.94, 17.03, $16.98,15.72$. Anal. Calcd for $\mathrm{C}_{39} \mathrm{H}_{52} \mathrm{O}_{3}$ : C, 82.35; H, 9.21. Found: C, 82.43; $\mathrm{H}, 9.27 \%$. MS: $\mathrm{m} / z$ [M-H] $]^{+}$found 567.28 $\left[\mathrm{C}_{39} \mathrm{H}_{52} \mathrm{O}_{3}\right]^{+}$requires 568.391. 
5'-Benzylfurano[3,2-b]olean-12-en-28-oic acid (20b). Colorless crystals (52\%). mp 96-98 ${ }^{\circ} \mathrm{C}$. $[\alpha]_{\mathrm{D}}^{19}+57.4^{\circ}(\mathrm{c}$ 1.09, $\left.\mathrm{CHCl}_{3}\right) .{ }^{1} \mathrm{H}$ NMR $\left(500 \mathrm{MHz}, \mathrm{CDCl}_{3}\right): \delta_{\mathrm{H}} 7.35-7.26(\mathrm{~m}, 5 \mathrm{H}$, arom), $5.67(\mathrm{~s}, 1 \mathrm{H}, \mathrm{H}-4$ '), $5.37(\mathrm{~m}, 1 \mathrm{H}, \mathrm{H}-12), 3.96$ (s, 2H, CH2-5'), $2.90(\mathrm{~m}, 1 \mathrm{H}, \mathrm{H}-18), 2.32\left(\mathrm{~d}, 1 \mathrm{H}, \mathrm{J} 15 \mathrm{~Hz}, \mathrm{H}^{\mathrm{a}}-1\right), 2.09\left(\mathrm{~m}, 1 \mathrm{H}, \mathrm{H}^{\mathrm{b}}-1\right), 2.03-1.25\left(\mathrm{~m}, 18 \mathrm{H}, \mathrm{CH}, \mathrm{CH}_{2}\right.$ in pentacyclic skeleton), 1.22, 1.17, 1.14, 1.01, 0.83 (all s, 3H each, H-23-H-27), 0.95 (s, 3H, H-30), 0.94 (s, 3H, H30). ${ }^{13} \mathrm{C}$ NMR $\left(125 \mathrm{MHz}, \mathrm{CDCl}_{3}\right): \delta_{\mathrm{C}} 184.82,155.12,152.29,143.44,138.75,128.76,128.38,126.25,122.78$, 113.58, 107.51, 53.50, 46.67, 46.22, 45.88, 41.74, 41.06, 39.41, 38.43, 38.08, 34.75, 34.55, 33.85, 33.11, 32.45, $32.06,30.71,29.29,27.76,25.81,23.60,23.38,22.95,21.53,18.99,16.86,15.56$. Anal. Calcd for $\mathrm{C}_{39} \mathrm{H}_{52} \mathrm{O}_{3}: \mathrm{C}_{\text {, }}$ 82.35; $\mathrm{H}, 9.21$. Found: $\mathrm{C}, 82.43 ; \mathrm{H}, 9.27 \%$. MS: $\mathrm{m} / \mathrm{z}[\mathrm{M}-\mathrm{H}]^{+}$, found $567.29\left[\mathrm{C}_{39} \mathrm{H}_{52} \mathrm{O}_{3}\right]^{+}$requires 568.39 .

\section{Acknowledgements}

The authors are deeply thankful to the staff members of the analytical center "Agidel" at the Institute of Petrochemistry and Catalysis of RAS for spectral measurement. This work was performed under financial support from the Russian Foundation for Basic Research (Project №. 16-33-6008).

\section{Supplementary Material}

Scans of the PMR and CMR spectra of all new compounds, and tabulated chemical shift data, are presented in the Supplementary file attached to this paper.

\section{References}

1. Cichewicz, R. H.; Kouzi, S. A. Med. Res. Rev. 2004, 24, 90.

http://dx.doi.org/10.1002/med.10053

2. Sarek, J.; Kvasnica, M.; Vlk, M.; Urban, M.; Dzubak, P.; Hajduch, M. In Research on Melanoma: A Glimpse into Current Directions and Future Trends; Murph, M. Eds.; In Tech: Rijeka, Croatia - European Union, 2011; Vol. 7, p 125. http://dx.doi.org/10.5772/19582

3. Mukherjee, R.; Kumar, V.; Srivastava, S. K.; Agarwal, S. K.; Burman, A. C. Anti-Cancer Agents Med. Chem. 2006, 6, 271.

http://dx.doi.org/10.2174/187152006776930846

4. Ali-Seyed, M.; Jantan, I.; Vijayaraghavan, K.; Bukhari, S. N. A. Chem. Biol. Drug Des. 2016, $87,517$. http://dx.doi.org/10.1111/cbdd.12682

5. Csuk, R. Expert Opin. Ther. Pat. 2014, 24, 1. http://dx.doi.org/10.1517/13543776.2014.927441

6. Zhang, D.-M.; Xu, H.-G.; Wang, L.; Li, Y.-J.; Sun, P.-H.; Wu, X.-M.; Wang, G.-J.; Chen, W.-M.; Ye, W.-C. Med. Res. Rev. 2015, 35, 1127.

http://dx.doi.org/10.1002/med.21353

7. Chen, H.; Gao, Y.; Wang, A.; Zhou, X.; Zheng, Y.; Zhou, J. Eur. J. Med. Chem. 2015, 92, 648. http://dx.doi.org/10.1016/j.ejmech.2015.01.031 
8. Lin, C.; Wen, X.; Sun, H. Expert Opin. Ther. Pat. 2016, 26, 643. http://dx.doi.org/10.1080/13543776.2016.1182988

9. Cui, H. - W.; He, Y.; Wang, J.; Gao, W.; Liu, T.; Qin, M.; Wang, X.; Gao, C.; Wang, Y.; Liu, M.-Y.; Yi, Z.; Qiu, W. Eur. J. Med. Chem. 2015, 95, 240. http://dx.doi.org/10.1016/j.ejmech.2015.03.048

10. Kvasnica, M.; Urban, M.; Dickinson, N. J.; Sarek, J. Nat. Prod. Rep. 2015, 32, 1303. http://dx.doi.org/10.1039/c5np00015g

11. Leal, A. S.; Wang, R.; Salvador, J. A. R.; Jing, Y. Org. Biomol. Chem. 2013, 11, 1726. http://dx.doi.org/10.1039/c3ob00011g

12. Zhang, H.; Zhu, F. Li, P.; Liu, J.; Yao, H.; Jiang, J.; Ye, W.; Wu, X.; Xu, J. Chem. Biol. Drug Des. 2015, 86, 424. http://dx.doi.org/10.1111/cbdd.12543

13. Borkova, L.; Adamek, R.; Kalina, P.; Drasar, P.; Dzubak, P.; Gurska, S.; Rehulka, J.; Hajduch, M.; Urban, M.; Sarek, J. Chem. Med. Chem. 2017, 12, 390. http://dx.doi.org/10.1002/cmdc.201600626

14. Xu, J.; Li, Z.; Luo, J.; Yang, F.; Liu, T.; Liu, M.; Qiu, W. -W.; Tang, J. J. Med. Chem. 2012, 55, 3122. http://dx.doi.org/10.1021/jm201540h

15. Wu, J.; Bao, B.-H.; Shen, Q.; Zhang, Y.-C.; Jiang, Q.; Li, J.-X. Med. Chem. Commun. 2016, 7, 371. http://dx.doi.org/10.1039/c5md00482a

16. Sousa, M. C.; Varandas, R.; Santos, R.C.; Santos, R. M.; Alves, V.; Salvador, J. A. R. Plos one 2014, 9 , e89939.

http://dx.doi.org/10.1371/journal.pone.0089939

17. Laavola, M.; Haavikko, R.; Hämäläinen, R.; Leppänen, T.; Nieminen, R.; Alakurtti, S.; Moreira, V. M.; YliKauhaluoma, J.; Moilanen, E. J. Nat. Prod. 2016, 79, 274.

http://dx.doi.org/10.1021/acs.jnatprod.5b00709

18. Haavikko, R.; Nasereddin, A; Sacerdoti-Sierra, N.; Kopelyanskiy, D.; Alakurtti, S.; Tikka, M.; Jaffe, Ch. L.; Kauhaluoma, J.Y. Med. Chem. Commun. 2014, 5, 445.

http://dx.doi.org/10.1039/c3md00282a

19. Banerjee, R.; Kumar, HKS.; Banerjee, M. Int. J. Rev. Life. Sci. 2012, 2, 7.

https://www.researchgate.net/publication/267423293

20. Lee, H.-K.; Chan, K.-F.; Hui, C.-W.; Yim, H.-K.; Wu, X.-W.; Wong, H. N. C. Pure Appl. Chem. 2005, 77, 139. http://dx.doi.org/10.1351/pac200577010139

21. Arcadi, A.; Marinelli, F.; Pini, E.; Rossi, E. Tetrahedron Lett. 1996, 37, 3387. http://dx.doi.org/10.1016/0040-40399600553-9

22. Vieser, R.; Eberbach, W. Tetrahedron Lett. 1995, 36, 4405. http://dx.doi.org/10.1016/0040-40399500785-B

23. Vitale, P.; Scilimati, A. Cur. Org. Chem. 2013, 17, 1986. http://dx.doi.org/10.2174/13852728113179990093

24. Kirsch, S. F. Org. Biomol. Chem. 2006, 4, 2076. http://dx.doi.org/10.1039/b602596j

25. Belting, V.; Krause, N. Org. Biomol. Chem. 2009, 7, 1221. http://dx.doi.org/10.1039/b819704k

26. Alonso, F.; Beletskaya, I. P.; Yus, M. Chem. Rev. 2004, 104, 3079. http://dx.doi.org/10.1021/cr0201068 
27. Chang, M.-Y.; Cheng, Y.-C.; Lu, Y.-J. Org. Lett. 2015, 17, 1264. http://dx.doi.org/10.1021/acs.orglett.5b00246

28. Spivak, A. Yu.; Gubaidullin, R. R.; Galimshina, Z. R.; Nedopekina, D. A.; Odinokov, V. N. Tetrahedron 2016, 72, 1249.

http://dx.doi.org/10.1016/j.tet.2016.01.024

29. Arcadi, A.; Rossi, E. Tetrahedron 1998, 54, 15253. http://dx.doi.org/10.1016/S0040-40209800953-3

30. Trofimov, B. A.; Schmidt, E. Yu. Russ. Chem. Rev. 2014, 83, 600. https://doi.org/10.1070/RC2014v083n07ABEH004425

31. Baldwin, J. E. J. Chem. Soc., Chem. Commun. 1976, 734. http://dx.doi.org/10.1039/C39760000734

32. Gilmore, K.; Alabugin, I. V. Chem. Rev. 2011, 111, 6513. http://dx.doi.org/10.1021/cr200164y

33. Kim, D. S. H. L.; Chen, Z.; Nguyen, T.; Pezzuto, J. M.; Qiu, Sh.; Lu, Z-Z. Synthetic. Commun. 1997, 9, 1607. http://dx.doi.org/10.1080/00397919708006099 